\title{
ATR-FTIR, FT-NIR and near-FT-Raman spectroscopic studies of molecular composition in human skin in vivo and pig ear skin in vitro
}

\author{
Tanja M. Greve ${ }^{\mathrm{a}, \mathrm{b}, *}$, Kristine B. Andersen ${ }^{\mathrm{a}}$ and Ole F. Nielsen ${ }^{\mathrm{b}}$ \\ ${ }^{a}$ Spectroscopy and Physical Chemistry, LEO Pharma A/S, 2750 Ballerup, Denmark \\ ${ }^{\mathrm{b}}$ Department of Chemistry, University of Copenhagen, 2100 Copenhagen, Denmark
}

\begin{abstract}
ATR-FTIR, FT-NIR and near-FT-Raman spectroscopy were used to characterize the molecular composition of human skin in vivo and pig ear skin in vitro. Due to different measurement depths the spectroscopic techniques reveal the characteristics of different layers of the skin. Tape stripping was used with the ATR-FTIR technique. Spectral differences concerning lipid content and conformation, protein secondary structure or content of water were found with respect to both gender and species (i.e. human versus pig ear) at all measured skin depths. New assignments of so far unassigned lipid and protein peaks in the FT-NIR and ATR-FTIR spectra of skin were made. PCA and PLS models were used to investigate the division of the recorded spectra into groups. With respect to classification of male and female subjects, the PLS discriminant analysis provided a classification accuracy of 64-93\% based on the ATR-FTIR spectra and 83-89\% based on the Raman spectra. With respect to classification of human skin in vivo and pig ear skin in vitro, the PLS discriminant analysis provided a classification accuracy of 75-100\% based on the Raman spectra and 100\% based on the ATR-FTIR spectra.
\end{abstract}

Keywords: Near-FT-Raman, ATR-FTIR, FT-NIR, human skin in vivo, pig ear skin in vitro, PCA

\section{Introduction}

Human skin is a complex multi-layered organ which acts as a barrier to prevent water loss and to protect the body from chemical and microbial attack. The skin essentially consists of three layers - the epidermis, dermis and the subcutaneous fatty layer. The barrier function of skin is mainly dependent on the stratum corneum (SC) which is a part of the epidermis and is the outermost layer of the skin. $\mathrm{SC}$ is only about $10-20 \mu \mathrm{m}$ thick and comprises approximately 15 layers [1]. Epidermis has a thickness of $75-150 \mu \mathrm{m}$ whereas dermis is in general $1-2 \mathrm{~mm}$ thick [1]. The hydration of the skin is approximately $20 \%$ in the upper layers of epidermis and increases to $70 \%$ (by weight) in the lower epidermal layers and throughout the dermis [1]. Proteins in the epidermis are keratins, which are predominantly in the $\alpha$-helical state [1,2] whereas proteins in the dermis are collagens, which have a triple-helical structure [3].

\footnotetext{
${ }^{*}$ Corresponding author: T.M. Greve, Spectroscopy and Physical Chemistry, LEO Pharma A/S, 2750 Ballerup, Denmark. Tel.: +45 7226 2912; Fax: +45 7226 3321; E-mail: tanja.greve@leo-pharma.com.
} 
Skin properties have been characterized by Raman and IR spectroscopy since the early 1990s [4]. Raman spectroscopy has been used for a molecular characterization of human stratum corneum samples [5,6] as well as full thickness skin in vitro [7,8] and in vivo [9]. Raman spectroscopy has further been used to address the characteristics of healthy and diseased skin [10-13] and to compare human and animal skin $[14,15]$. IR spectroscopy has been a tool for the general characterization of the stratum corneum [16,17] and with the use of the Attenuated Total Reflectance (ATR) technique IR spectroscopy has become greatly used in the skin absorption studies of various chemicals and formulations [18-23].

NIR spectroscopy has predominantly been applied for skin characterizations with respect to water content [24-30].

Only a few studies tend to distinguish male and female skin [31-33] and these studies are either performed on too few subjects or do not find the results specific enough to assign the observed similarities and dissimilarities to the components of the skin. Apart from skin studies, Raman spectroscopy was recently used to classify human gender from finger nails [34] but mainly from a chemometric point of view and with very few comments on the spectral findings or the relation to the skin components.

In this study we present the combination of Raman, NIR and ATR-FTIR spectroscopy to characterize all skin layers. Male and female skin in vivo is compared from a spectroscopic as well as a chemometric point of view with respect to gender and age. A comparison is performed with pig ear skin in vitro which is a well-accepted and readily available model for the human barrier often used to assess topical and transdermal pharmaceuticals in vitro [35].

\section{Materials and methods}

\subsection{Skin samples}

In vivo spectra were recorded from a total of 92 volunteers (40 males and 52 females) at the age of 20-65 years (the average age was 37 years for females and 36 years for males). Informed consent was obtained from all subjects. The study was approved by The Danish National Committee on Biomedical Research Ethics. Forty-two volunteers were measured with all three spectroscopic techniques whereas the others were only measured using one or two different techniques due to instrumental problems during the measurement period. Thus, Raman spectra were collected from 28 males and 32 females, ATR-FTIR spectra were collected from 22 males and 31 females and NIR spectra were collected from 32 males and 49 females. All measurements were performed on the left ventral forearm because this is a relatively hair-free site, although a few male subjects were shaved prior to measurements. No other pre-treatment was performed on the sites.

In vitro spectra were recorded from 73 pig ears. Due to conservation reasons and keeping the ears as fresh as possible it was decided that the same ear should not be used for all three techniques. Therefore Raman and NIR spectra were recorded of 50 pig ears, whereas the last 23 pig ears were used for ATR-FTIR measurements. The pig ears were obtained from a local abattoir within a few hours postmortem. The skin surface was washed, dried and shaved using an animal clipper (Golden A5 from Oster, Tennessee, USA). The spectroscopic measurements were performed without further treatment of the membrane (i.e., the skin remained on the isolated ear).

\subsection{Tape stripping}

The ATR-FTIR spectra of the 53 human skin samples in vivo and the 23 pig ear skin samples in vitro were recorded following a sequential tape stripping procedure [36]. This technique uses standard adhe- 
sive tape (type Q-connect, from Interaction Belgium) to remove a layer of stratum corneum with each sequentially applied piece of tape leaving the skin approximately $0.5 \mu \mathrm{m}$ thinner [37]. An ATR-FTIR spectrum was recorded of the skin prior to tape stripping. Hereafter a piece of tape was applied to the skin for $20 \mathrm{~s}$ then stripped off and an ATR-FTIR spectrum was recorded again. This sequence was applied for 4 tape-strips. Subsequently, spectra were recorded every two strips up to a total of 16 strips. Thus, 11 spectra were recorded on each skin sample, giving a total of 836 ATR-FTIR spectra. The time delay between each sequentially recorded spectrum is $3 \mathrm{~min}$ and $20 \mathrm{~s}$ for the first 4 tape strips and $3 \mathrm{~min}$ and $40 \mathrm{~s}$ for the following strips.

\subsection{Separation of skin layers}

For measurements of different skin layers in pig ear skin samples, epidermis and dermis was separated by heat separation [38]; a $3 \times 3 \mathrm{~cm}$ skin biopsy was cut off the ear, subcutaneous fat was removed using a scalpel and the skin was placed in a dessiccator over condensed water steam at $60^{\circ} \mathrm{C}$. After $5 \mathrm{~min}$ the intact epidermis inclusive SC was gently teased off with forceps which gives epidermal samples of approximately $200 \mu \mathrm{m}$ thickness and dermal samples of approximately $2 \mathrm{~mm}$. During the spectral measurements all samples were placed on stainless steel plates, which do not give rise to any bands in the Raman spectra.

\subsection{ATR-FTIR spectroscopy}

ATR-FTIR spectra were recorded on a Bruker Equinox 55 spectrometer equipped with a DLATGS detector. The spectrometer was coupled with an MKII Golden Gate ${ }^{\text {TM }}$ Single Reflection ATR System (Specac), which has a diamond crystal of approximately $1 \mathrm{~mm}$ in diameter. The spectral range of the acquired spectra was $4000-650 \mathrm{~cm}^{-1}$. For each sample 200 spectra at $5 \mathrm{~cm}^{-1}$ resolution were collected for $3 \mathrm{~min}$ and averaged. The penetration depth $\left(d_{\mathrm{p}}\right)$ of the IR radiation is given by [39]:

$$
d_{\mathrm{p}}=\frac{\lambda}{2 \pi \cdot n_{2} \cdot\left(\sin ^{2} \theta-\left(n_{1} / n_{2}\right)^{2}\right)^{1 / 2}},
$$

where $\lambda$ is the wavelength; $\theta$ is the angle of incidence of the IR beam into the sample; $n_{1}$ is the refractive index of the sample and $n_{2}$ is the refractive index of the ATR crystal. The specification for the Golden Gate ATR system gives an incident angle of $45^{\circ}$ and a refractive index of 2.4. The refractive index of skin is 1.55 [40] and hence the penetration depth in the spectral range is $0.6-3.5 \mu \mathrm{m}$. This means that only vibrations from molecules in the outermost layers of the stratum corneum can be seen in the spectra. By the tape stripping procedure each spectrum contains information about new skin layers within the stratum corneum.

\subsection{FT-NIR spectroscopy}

A Bruker Equinox 55 spectrometer equipped with a DLATGS detector was used to obtain FT-NIR spectra in the region $4000-400 \mathrm{~cm}^{-1}$. The spectrometer was coupled with a fiber-optic probe which has a circular measurement area of approximately $4 \mathrm{~mm}$ in diameter. For each sample 64 scans at $8 \mathrm{~cm}^{-1}$ resolution were collected for $1 \mathrm{~min}$ and averaged. The measurements are based on diffuse reflectance. The measurement depth was reported to 150-200 $\mu \mathrm{m}$ [41]. However, there are also reports using the 50\% measurement depth (i.e. half of all the absorbed energy is absorbed above this depth) which is $120 \mu \mathrm{m}$ 
at $6700 \mathrm{~cm}^{-1}$ and $30 \mu \mathrm{m}$ at $5250 \mathrm{~cm}^{-1}$ [30]. Yet another report states that the penetration depth of the incident NIR light is up to $2 \mathrm{~mm}$ but due to scattering the majority of the light returning from the skin and contributing to the spectra will come from the upper layers of the skin, as that fraction of light will be the least attenuated [26]. Thus, although the arguments differ and the exact statements of the depth vary, it is agreed that the measurement depth is wavelength dependent but within the epidermal layers of the skin.

\subsection{Near-FT-Raman spectroscopy}

A Bruker RFS 100/S Raman spectrometer was used, equipped with a nitrogen cooled Ge-detector and a Nd:YAG-laser providing a power of $1500 \mathrm{~mW}$. The spectrometer was coupled with a fibre optic probe. During measurements the probe head was placed in contact with the skin, the laser was delivered to the tissue via an optical fibre and the scattered radiation was returned to the spectrometer via a fibre bundle. Due to loss of power to the fibres, the actual laser power at the sample surface was approximately $900 \mathrm{~mW}$. The spectral range of the acquired spectra was $3500-250 \mathrm{~cm}^{-1}$. For each sample 500 scans at $4 \mathrm{~cm}^{-1}$ resolution were collected for $15 \mathrm{~min}$ and averaged. The measurement depth of the Raman spectrometer is up to several hundred micrometers [9], which is beyond the boundary of epidermis but within the boundary of dermis. Hence each spectrum is in essence the average spectrum across the various skin layers.

\subsection{Curve fitting}

Prior to curve fitting the data were converted to ASCII files and curves were then fitted using Origin Pro 7.5 software (OriginLab Corporation). We used the Lorentzian band shape as this gave the best fit. The appropriate number of bands for the curve fitting procedure was determined from the second derivative of the spectra. The program fitted a linear baseline to each spectrum and then used iterations to find the best combination of heights, positions and widths that fitted the data.

The Raman spectra were normalized by use of the band at approximately $2940 \mathrm{~cm}^{-1}$. This predominantly protein band is relatively unaffected by the protein secondary structure and hence are expected to show similar intensities in all skin samples.

The O-H bending vibration band at $1640 \mathrm{~cm}^{-1}$ is relatively stronger in the IR-spectra than in the Raman spectra. Thus this band interferes with the amide I bands in the ATR-FTIR spectra. In order to avoid this, a water spectrum was subtracted prior to curve fitting. The spectrum subtraction was performed to suppress the intensity of the $\mathrm{O}-\mathrm{H}$ stretching vibration mode around $3400 \mathrm{~cm}^{-1}$. Examples of this curve fitting procedure were recently reported by us [23].

\subsection{Chemometrics}

Prior to multivariate data analysis all spectral data sets were converted to JCAMP files and pretreated with multiplicative scatter correction (MSC) to avoid differences in baseline offset. The Raman spectra were further baseline corrected to eliminate background scattering.

The Unscrambler ver. 8.0 (CAMO, Norway) was used for the PCA and PLS models. 


\section{Results and discussion}

There are no visually detectable differences or gradual changes in the spectra with respect to age. Age will therefore only be used as a variable in the chemometrics section. The ability of analyzing such a gradual change across all the recorded spectra is one of the advantages of the chemometric tools.

\subsection{Near-FT-Raman spectra}

Raman spectra were recorded from 29 males, 31 females and 50 pig ears. An average of the spectra for each group is given in Fig. 1. Prior to further analysis the spectra are divided into smaller regions with different characteristic features: the $3500-2800 \mathrm{~cm}^{-1}$ region contains the $\mathrm{O}-\mathrm{H}$ stretching vibration band from water around $3220 \mathrm{~cm}^{-1}[9,42,43]$ and the $\mathrm{C}-\mathrm{H}$ stretching vibration bands of which the bands at 2985 and $2938 \mathrm{~cm}^{-1}$ are predominantly from the skin proteins whereas the 2885 and $2856 \mathrm{~cm}^{-1}$ are from the skin lipids $[5,10,44]$. The $1780-1580 \mathrm{~cm}^{-1}$ region contains the $\mathrm{C}=\mathrm{O}$ stretching vibration band from lipid esters at $1750 \mathrm{~cm}^{-1}$ [4] and the amide I bands from proteins around $1650 \mathrm{~cm}^{-1}$ composed of several overlapping bands characteristic of different secondary structures [45]. Band assignments are given in Table 1. The 1365-1215 $\mathrm{cm}^{-1}$ region contains 5 overlapping bands, of which the two around 1340 and $1320 \mathrm{~cm}^{-1}$ are assigned to $\mathrm{CH}_{2}$ wagging and twisting vibrations, respectively, of proteins in the epidermis (i.e. keratins). The one around $1300 \mathrm{~cm}^{-1}$ is assigned to the $\mathrm{CH}_{2}$ twisting vibration of lipids [5] and the last two around 1270 and $1245 \mathrm{~cm}^{-1}$ are the amide III bands from proteins with different secondary structures [4].

Curve fitting procedures reveal the peak positions and areas in Table 1. The individual peaks are found at the same wavenumber positions for the various skin types - except for the $1750 \mathrm{~cm}^{-1}$ that in general could not be observed in the pig ear spectra. The areas of the individual bands show significant gender variation and the in vitro pig ear areas significantly differ from the human in vivo band areas. In vivo spectra of female skin have larger peak areas around 2885, 2855, 1750, $1300 \mathrm{~cm}^{-1}$ than does the in

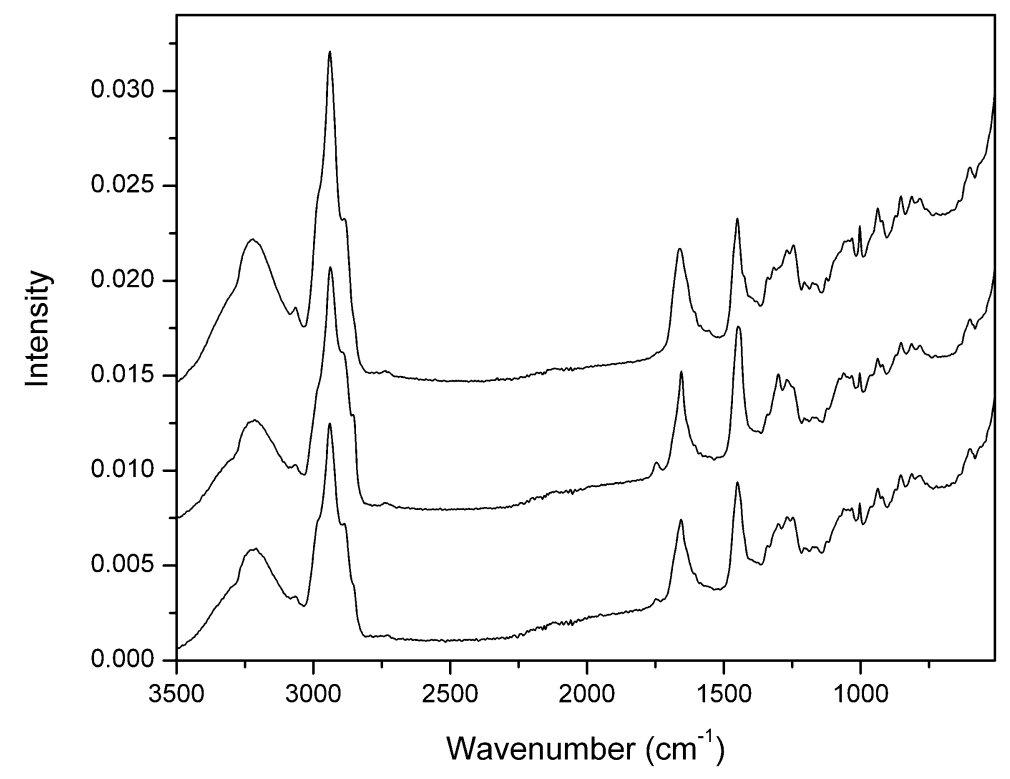

Fig. 1. Average Raman spectra of male (bottom) and female (middle) skin in vivo and pig ear skin samples in vitro (top). 
Table 1

Assignments (following the comments in the text), peak position and areas of selected bands in the Raman spectra of male and female in vivo and pig ear in vitro skin spectra. Values given as mean \pm standard deviation

\begin{tabular}{|c|c|c|c|c|c|c|}
\hline \multirow[t]{2}{*}{ Assignment } & \multicolumn{3}{|c|}{ Wavenumbers $\left(\mathrm{cm}^{-1}\right)$} & \multicolumn{3}{|c|}{ Areas } \\
\hline & Female & Male & Pig ears & Female & Male & Pig ears \\
\hline$\overline{\nu(\mathrm{OH}), \text { water }}$ & $3217 \pm 2$ & $3218 \pm 1$ & $3218 \pm 2$ & $112.9 \pm 20.4$ & $128.2 \pm 20.1$ & $128.8 \pm 23$ \\
\hline$\nu_{\text {as }}\left(\mathrm{CH}_{3}\right)$, protein & $2985 \pm 2$ & $2986 \pm 1$ & $2984 \pm 2$ & $15.6 \pm 2.2$ & $15.4 \pm 1.6$ & $15.8 \pm 3.0$ \\
\hline$\nu_{\mathrm{s}}\left(\mathrm{CH}_{3}\right)$, protein & $2937 \pm 2$ & $2939 \pm 1$ & $2938 \pm 2$ & $78.2 \pm 1.8$ & $77.7 \pm 1.5$ & $76.8 \pm 3.2$ \\
\hline$\nu_{\mathrm{S}}\left(\mathrm{CH}_{2}\right)$, lipid & $2886 \pm 1$ & $2885 \pm 1$ & $2882 \pm 2$ & $24.0 \pm 5.2$ & $17.2 \pm 3.6$ & $13.7 \pm 2.2$ \\
\hline$\nu_{\text {as }}\left(\mathrm{CH}_{2}\right)$, lipid & $2858 \pm 3$ & $2855 \pm 4$ & $2857 \pm 8$ & $4.0 \pm 2.3$ & $1.4 \pm 1.0$ & $0.4 \pm 0.2$ \\
\hline$\nu(\mathrm{C}=\mathrm{O})$, lipid & $1747 \pm 4$ & $1750 \pm 3$ & & $1.5 \pm 0.5$ & $0.9 \pm 0.5$ & \\
\hline$\nu(\mathrm{C}=\mathrm{O})$, amid $\mathrm{I}, \beta$-sheet & $1687 \pm 4$ & $1687 \pm 4$ & $1687 \pm 3$ & $1.3 \pm 0.7$ & $2.0 \pm 1.0$ & $2.0 \pm 1.0$ \\
\hline$\nu(\mathrm{C}=\mathrm{O})$, amid $\mathrm{I}, \beta$-sheet or collagen & $1673 \pm 3$ & $1672 \pm 4$ & $1672 \pm 4$ & $3.5 \pm 1.1$ & $4.4 \pm 1.0$ & $5.3 \pm 1.8$ \\
\hline$\nu(\mathrm{C}=\mathrm{O})$, amid $\mathrm{I}, \alpha$-helix & $1655 \pm 1$ & $1656 \pm 2$ & $1657 \pm 3$ & $13.5 \pm 2.1$ & $10.9 \pm 2.3$ & $8.7 \pm 2.3$ \\
\hline$\nu(\mathrm{C}=\mathrm{O})$, amid $\mathrm{I}, \beta$-sheet or collagen & $1632 \pm 3$ & $1635 \pm 3$ & $1635 \pm 3$ & $2.9 \pm 0.8$ & $4.7 \pm 1.4$ & $5.2 \pm 1.5$ \\
\hline$\nu(\mathrm{C}=\mathrm{O})$, amid $\mathrm{I}$ & $1608 \pm 6$ & $1610 \pm 6$ & $1609 \pm 4$ & $0.6 \pm 0.5$ & $0.8 \pm 0.6$ & $0.6 \pm 0.4$ \\
\hline$\delta\left(\mathrm{CH}_{2}\right)$, protein & $1342 \pm 2$ & $1342 \pm 2$ & $1341 \pm 2$ & $0.8 \pm 0.3$ & $1.1 \pm 0.3$ & $1.3 \pm 0.4$ \\
\hline$\delta\left(\mathrm{CH}_{2}\right)$, protein & $1319 \pm 3$ & $1319 \pm 3$ & $1320 \pm 2$ & $2.1 \pm 0.7$ & $2.7 \pm 1.1$ & $2.6 \pm 0.7$ \\
\hline$\delta\left(\mathrm{CH}_{2}\right)$, lipid & $1301 \pm 1$ & $1300 \pm 3$ & $1299 \pm 4$ & $7.2 \pm 1.9$ & $4.5 \pm 1.9$ & $2.1 \pm 0.8$ \\
\hline$\nu(\mathrm{CN})$, amid III, $\alpha$-helix & $1268 \pm 2$ & $1270 \pm 2$ & $1271 \pm 2$ & $7.9 \pm 1.2$ & $7.3 \pm 1.2$ & $5.7 \pm 1.3$ \\
\hline$\nu(\mathrm{CN})$, amid III, disordered & $1245 \pm 2$ & $1245 \pm 1$ & $1245 \pm 1$ & $3.1 \pm 1.0$ & $3.9 \pm 0.8$ & $4.0 \pm 1.0$ \\
\hline
\end{tabular}

vivo male and in vitro pig ear skin spectra. These peaks are all assigned to the lipid content of the skin and hence it is shown that female skin contains more lipids than male or pig ear skin. When comparing female and pig ear skin only, further differences are found at 3218,1673, 1632 and $1245 \mathrm{~cm}^{-1}$, where the pig ear skin spectra show larger peak areas for each peak. The $3218 \mathrm{~cm}^{-1}$ peak is assigned to water and the others are assigned to either epidermal keratins with a $\beta$-sheet structure [44] or to the collagens in dermis [3] and hence it is shown that pig ear skin contains more water and more proteins that are not the epidermal $\alpha$-keratins. Unfortunately, the bands around 1673 and $1632 \mathrm{~cm}^{-1}$ cannot be specifically assigned to either $\beta$-keratin or collagen, because both proteins are reported to give amide I bands around 1670 and $1640-1630 \mathrm{~cm} \mathrm{[3,47].}$

Pig ear skin is described to be generally of the same thickness as human skin with respect to SC and epidermis [35]. However, male skin is described to be thicker than female skin [48] and the differences found between male, female and pig ear skin spectra may in part be explained by differences in skin thicknesses. In the thinner female skin, the measurement depth is beyond the boundary of the dermis and thus the subcutaneous fat contributes to the spectra. For the thicker male skin the spectra contain only a small contribution from the subcutaneous fat but a larger contribution from the collagens in dermis. But differences in skin thicknesses do not account for all spectral differences: the 1340, 1320, 1270 and $1245 \mathrm{~cm}^{-1}$ bands are assigned to proteins in general [4]. By separating epidermis and dermis in pig ear skin samples (Fig. 2) we find, that the bands at 1340 and $1320 \mathrm{~cm}^{-1}$ are predominantly from the epidermal keratins whereas the bands at 1270 and $1245 \mathrm{~cm}^{-1}$ are predominantly from the dermal collagens. This assignment is supported by previously reported spectra [7]. Hence the 1340 and $1320 \mathrm{~cm}^{-1}$ bands are most likely assigned to the amid III bands of $\alpha$-keratin, since $\alpha$-helices in general show amide III bands at $1320-1260 \mathrm{~cm}^{-1}$ [45]. However, the lipids also contribute to the $1270 \mathrm{~cm}^{-1}$ band [6,7] and hence this band cannot solely be assigned to the proteins in dermis. The 1340,1320 and 


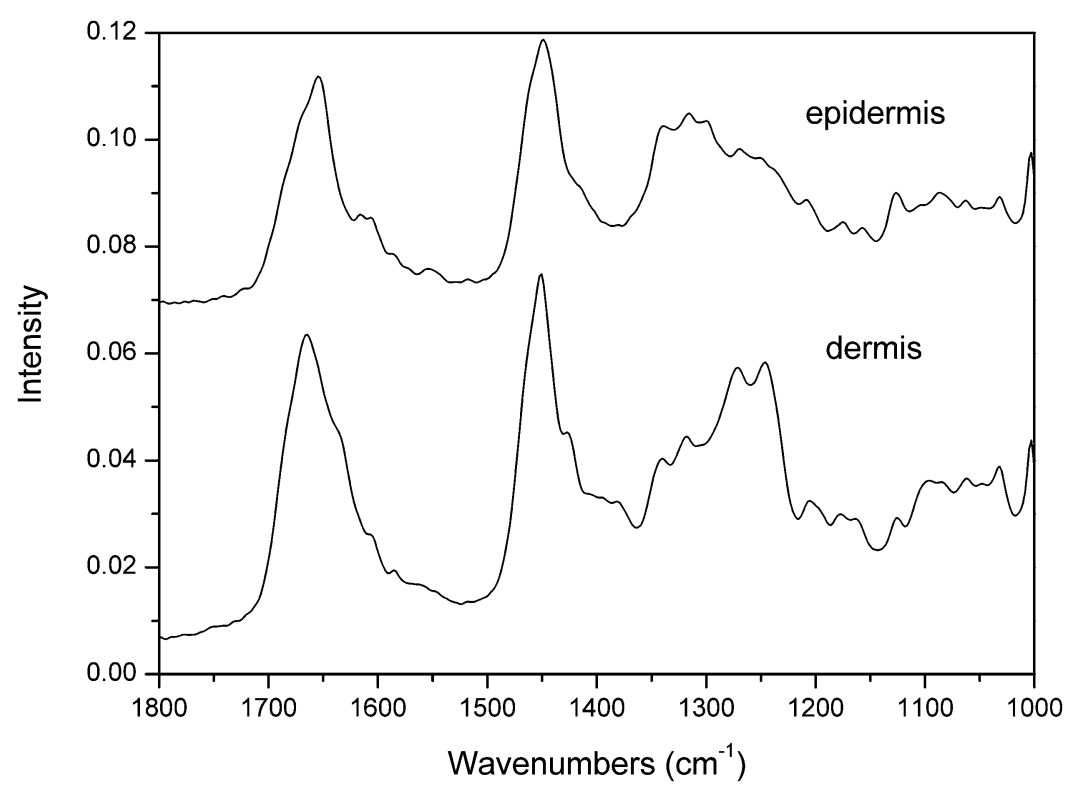

Fig. 2. Raman spectra of epidermis and dermis from pig ear skin in vitro.

$1245 \mathrm{~cm}^{-1}$ band areas are on an average the same in the male and pig ear spectra and therefore both the epidermal and the dermal skin layer must be of the same thickness for male and pig ear skin. The higher intensity of the 2855,1750 and $1300 \mathrm{~cm}^{-1}$ bands in the male spectra show that male skin relative to pig ear skin shows higher lipid content. Since the male and pig ear skin layers are of the same thicknesses, the increased amount of lipids can not be explained by an increased contribution from the subcutaneous layer but must be found within the skin. Likewise, because the male and pig ear skin thicknesses are equal the relative increase of the areas of $\beta$-keratin or collagen bands in pig ear skin must arise from variations within the skin as well. When comparing male and female skin spectra the 1340, 1320 and $1245 \mathrm{~cm}^{-1}$ band areas are smaller in the female spectra and hence the variations with respect to gender in this spectral region corroborates the known differences in skin thicknesses. The 1340, 1320 and 1245 $\mathrm{cm}^{-1}$ band areas are all approximately $23 \%$ increased in the male skin spectra compared to the female skin spectra, hence showing that the epidermal and dermal layers are both approximately $23 \%$ thicker in male skin. Thus all other spectral signals assigned to either layer should have a $23 \%$ percentage increase in the male skin spectra compared to female skin spectra. The $3200 \mathrm{~cm}^{-1}$ water band is only approximately $12 \%$ increased in the male skin spectra compared to female skin, hence showing that male skin must contain a relatively lower amount of water than female skin, which is supported by previously reported findings [33]. The 1673 and $1632 \mathrm{~cm}^{-1}$ bands - assigned to either $\beta$-keratin or collagen or a combination of both - are approximately $35 \%$ increased in the male skin spectra compared to female skin spectra, which is more than the increase in skin thickness, hence showing a higher amount of proteins with those structures in male skin than in female skin.

\subsection{FT-NIR spectra}

Average NIR spectra of 32 male and 49 female in vivo and 50 pig ear in vitro skin spectra are shown in Fig. 3. The dominating features of the spectra are the two water peaks at 6900 and $5180 \mathrm{~cm}^{-1}$ assigned to the first overtone of the $\mathrm{OH}$ stretching vibration and the combination mode of the $\mathrm{OH}$ stretching and 


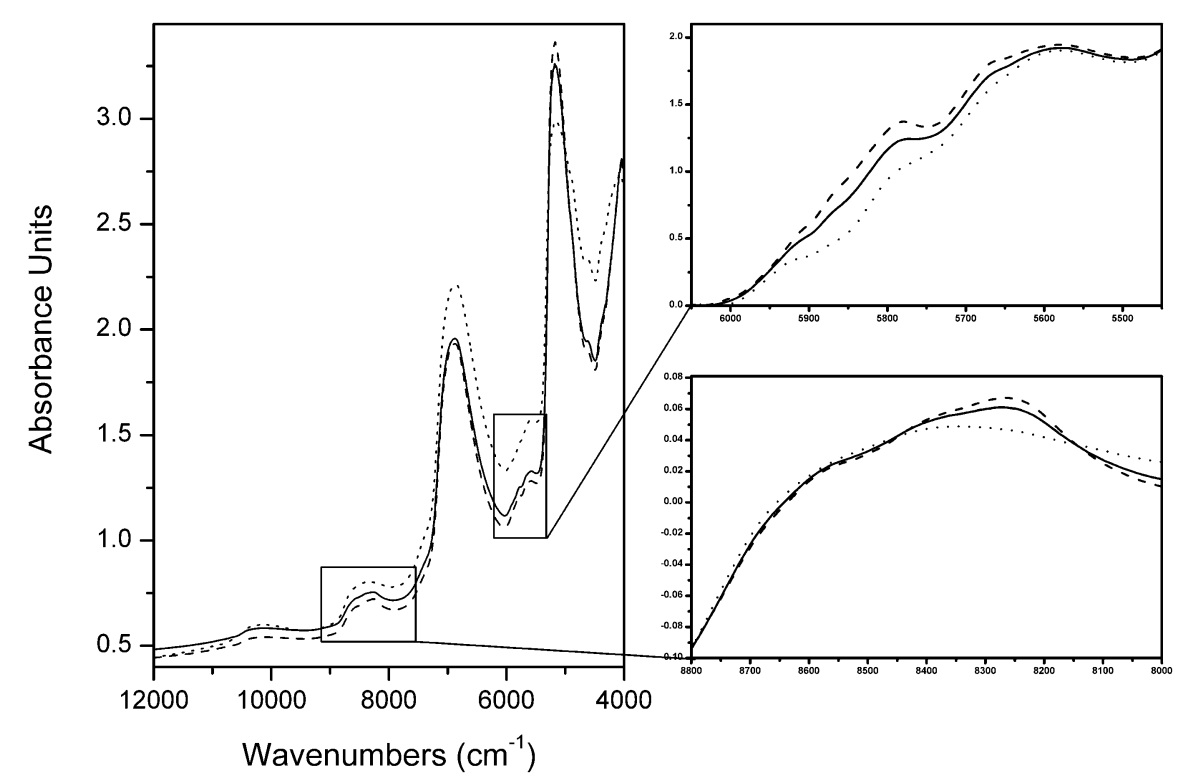

Fig. 3. Average NIR spectra of male and female skin samples, in vivo, and pig ear skin samples, in vitro.

$\mathrm{HOH}$ bending vibrations [24]. The $5180 \mathrm{~cm}^{-1}$ band consists of several overlapping bands assigned to protein-bound water, bulk water and water associated with the lipid bilayers [25]. There are no specific assignments of the $6900 \mathrm{~cm}^{-1}$ band. A correlation of water intensity variations and skin types cannot be performed.

Weaker overlapping bands are found at 8550, 8410, 8220, 5925, 5780, 5675 and $5600 \mathrm{~cm}^{-1}$ (Fig. 3). The 5780 and $5600 \mathrm{~cm}^{-1}$ bands are assigned to $\mathrm{CH}$ groups in skin lipids and proteins, respectively [25]. No assignments have been found for the remaining bands. Comparison of the male, female and pig ear skin spectra in the $8800-8000$ and $6050-5450 \mathrm{~cm}^{-1}$ regions shows great variations, as can be seen in Fig. 3. For each of the bands at $8410,8220,5925,5780$ and $5675 \mathrm{~cm}^{-1}$ the female spectra show the highest peak intensities and the pig ear skin spectra the lowest peak intensities. The male spectra show intensities in between the two. The $5780 \mathrm{~cm}^{-1}$ band was assigned to skin lipids [25] and according to the intensity variations it seems justified to assign the bands at $8410,8220,5925$ and $5675 \mathrm{~cm}^{-1}$ to skin lipids as well. All skin spectra in Fig. 3 show essentially the same intensities of the 8550 and $5600 \mathrm{~cm}^{-1}$ bands. The latter was assigned to skin proteins [25] and due to the similar intensity variations for the two bands the $8550 \mathrm{~cm}^{-1}$ band is also assigned to skin proteins.

The NIR spectra show that in the epidermal layers of the skin, females have a greater amount of lipids as compared to male and pig ears, whereas all skin types have the same content of proteins. The NIR spectra hence corroborates the Raman spectroscopic results in showing that of the three skin types male, female and pig ear skin - female in vivo and pig ear in vitro skin spectra differs the most whereas male spectra are found somewhere in between.

\subsection{ATR FT-IR spectra}

Average ATR FT-IR spectra of 22 male and 31 female in vivo and 23 pig ear in vitro skin spectra are shown in Fig. 4. The spectra are recorded after 2 tape strips as the first spectra in the sequence may be influenced by factors from outside the skin. The ATR technique is very sensitive to the contact 


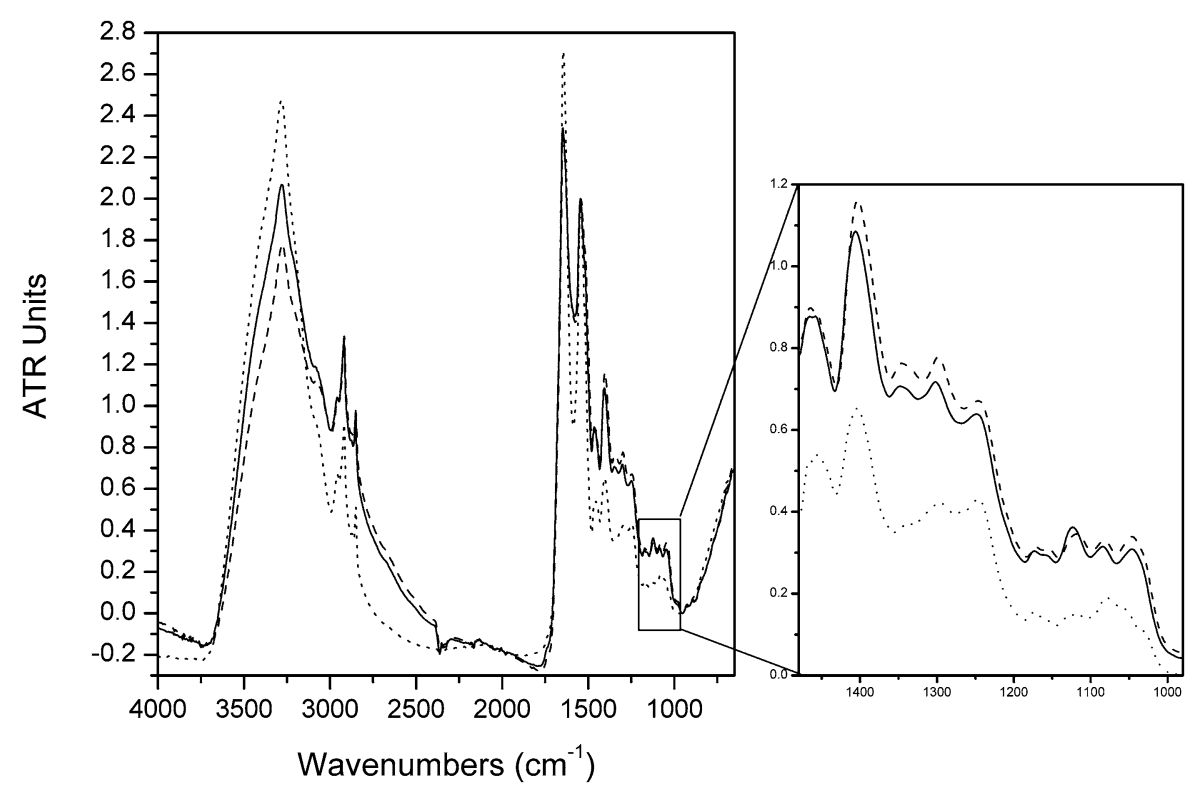

Fig. 4. Average ATR FT-IR spectra of female (solid), male (dotted) and pig ear (dashed) skin spectra. Spectra recorded after 2 tape strips.

state during the measurements and the overall intensity of the spectra varies a lot. Hence it is necessary to normalize the spectra. The most intense protein bands are the amide I and II bands at 1650 and $1540 \mathrm{~cm}^{-1}$, respectively, but these are overlapping with the $\mathrm{OH}$ water bending vibration at $1640 \mathrm{~cm}^{-1}$ and none of them are ideal as internal standards. However, the spectra were normalized after the amide II band since this is least influenced by water. Obviously the intensities of the water bands at $3400 \mathrm{~cm}^{-1}$ vary between species. The intensity is strongest in the pig ear spectra and weakest in the female spectra. Figure 4 shows in some details spectral variations in the region from 1480 to $1010 \mathrm{~cm}^{-1}$.

All spectra recorded after 2 tape strips were curve fitted in the 1710-1590, 1480-1215 and 1185$1010 \mathrm{~cm}^{-1}$ regions. Table 2 shows band assignments, fitted peak positions and relative percentages of the band areas. In calculations of the relative content of different secondary structures an average of the two bands at 1679 and $1630 \mathrm{~cm}^{-1}$, assigned to keratins with a $\beta$-sheet secondary structure, was used $[23,46,47]$. The secondary protein structures determined from areas of the four overlapping bands in the amide I region at $1710-1590 \mathrm{~cm}^{-1}$ are very similar for female, male and pig ears skin. The content is approximately $63 \% \alpha$-helix, $23 \% \beta$-sheet and $14 \%$ turns for each of the three groups.

In the 1480-1215 $\mathrm{cm}^{-1}$ region, the bands at 1465 and $1455 \mathrm{~cm}^{-1}$ are assigned to the $\mathrm{CH}_{2}$ bending vibrations of lipids and proteins, respectively $[4,45]$. A reliable curve fitting of these two closely overlapping bands is not possible and the calculated areas must be analyzed in combination with a visual inspection. Figure 4 shows that the lipid band at $1465 \mathrm{~cm}^{-1}$ varies in intensity relative to the protein band at $1455 \mathrm{~cm}^{-1}$ for the different skin types. The protein band is the more intense in the pig ear spectra whereas the lipid band is the more intense in the female spectra and in the male spectra the bands have equal intensities. The amide III band at $1245 \mathrm{~cm}^{-1}$ shows similar intensity for the three groups corroborating a very similar content of proteins and a varying content of lipids with the female group showing the highest content. A large variation in intensity is observed for the $1340 \mathrm{~cm}^{-1}$ band, which has previously been reported, although no assignment was given $[4,6]$. This band is barely seen in the pig ear skin spectra, whereas it is relatively intense in the female skin spectra and of medium intensity in 
Table 2

Assignments $[4,6,46]$, peak position and percentage areas of selected bands in the ATR IR spectra of male and female in vivo and pig ear in vitro skin, which have been tape stripped twice. Values are given as mean \pm standard deviation

\begin{tabular}{|c|c|c|c|c|c|c|}
\hline \multirow[t]{2}{*}{ Assignment } & \multicolumn{3}{|c|}{ Wavenumbers $\left(\mathrm{cm}^{-1}\right)$} & \multicolumn{3}{|c|}{ Areas $(\%)$} \\
\hline & Female & Male & Pig ears & Female & Male & Pig ears \\
\hline$\nu(\mathrm{C}=\mathrm{O})$, amid $\mathrm{I}, \beta$ & $1679 \pm 1$ & $1678 \pm 2$ & $1679 \pm 1$ & $4.8 \pm 0.8$ & $5.0 \pm 1.1$ & $5.3 \pm 0.8$ \\
\hline$\nu(\mathrm{C}=\mathrm{O})$, amid $\mathrm{I}$, turns & $1664 \pm 1$ & $1664 \pm 2$ & $1664 \pm 1$ & $11.8 \pm 0.9$ & $11.9 \pm 1.1$ & $12.9 \pm 1.4$ \\
\hline$\nu(\mathrm{C}=\mathrm{O})$, amid $\mathrm{I}, \alpha$ & $1648 \pm 0$ & $1648 \pm 1$ & $1647 \pm 1$ & $49.4 \pm 1.9$ & $49.4 \pm 2.2$ & $50.6 \pm 2.5$ \\
\hline$\nu(\mathrm{C}=\mathrm{O})$, amid $\mathrm{I}, \beta$ & $1630 \pm 0$ & $1630 \pm 0$ & $1630 \pm 1$ & $34.0 \pm 1.4$ & $33.6 \pm 1.4$ & $31.2 \pm 1.4$ \\
\hline$\delta\left(\mathrm{CH}_{2}\right)$, lipid & $1466 \pm 1$ & $1465 \pm 1$ & $1466 \pm 0$ & $4.3 \pm 0.9$ & $4.0 \pm 0.6$ & $2.3 \pm 0.7$ \\
\hline$\delta\left(\mathrm{CH}_{2}\right)$, protein & $1455 \pm 1$ & $1454 \pm 1$ & $1453 \pm 1$ & $2.3 \pm 0.8$ & $3.8 \pm 2.0$ & $9.2 \pm 2.2$ \\
\hline \multirow[t]{2}{*}{$\delta\left[\mathrm{C}\left(\mathrm{CH}_{3}\right)_{2}\right] \mathrm{sym}$} & $1399 \pm 1$ & $1400 \pm 1$ & $1402 \pm 1$ & $45.0 \pm 1.4$ & $44.5 \pm 2.5$ & $42.3 \pm 2.2$ \\
\hline & $1339 \pm 1$ & $1340 \pm 3$ & $1334 \pm 4$ & $9.2 \pm 3.7$ & $6.1 \pm 4.1$ & $0.9 \pm 0.6$ \\
\hline$\delta\left(\mathrm{CH}_{2}\right)$, lipid & $1295 \pm 1$ & $1297 \pm 2$ & $1294 \pm 2$ & $24.7 \pm 1.4$ & $24.2 \pm 2.2$ & $25.0 \pm 1.5$ \\
\hline$\nu(\mathrm{CN})$, amid III & $1245 \pm 1$ & $1245 \pm 1$ & $1245 \pm 1$ & $14.3 \pm 2.0$ & $17.4 \pm 4.5$ & $19.5 \pm 1.4$ \\
\hline \multirow[t]{2}{*}{$\nu(\mathrm{CC}), \delta(\mathrm{COH})$} & $1170 \pm 1$ & $1170 \pm 1$ & $1171 \pm 1$ & $1.4 \pm 0.5$ & $1.6 \pm 0.8$ & $2.9 \pm 0.8$ \\
\hline & $1156 \pm 3$ & $1157 \pm 3$ & $1159 \pm 3$ & $3.3 \pm 1.8$ & $2.4 \pm 1.7$ & $1.1 \pm 0.6$ \\
\hline \multirow[t]{3}{*}{$\nu(\mathrm{CC})$, lipid, trans } & $1118 \pm 2$ & $1120 \pm 2$ & $1120 \pm 2$ & $23.6 \pm 3.7$ & $29.8 \pm 6.3$ & $14.7 \pm 4.7$ \\
\hline & $1081 \pm 1$ & $1080 \pm 1$ & $1079 \pm 1$ & $28.9 \pm 4.1$ & $29.0 \pm 5.1$ & $46.7 \pm 6.6$ \\
\hline & $1048 \pm 1$ & $1046 \pm 2$ & $1052 \pm 1$ & $32.2 \pm 3.9$ & $29.0 \pm 4.3$ & $28.2 \pm 4.1$ \\
\hline$\nu(\mathrm{CC})$, lipid, cis & $1031 \pm 1$ & $1031 \pm 1$ & $1029 \pm 1$ & $10.5 \pm 1.3$ & $8.3 \pm 1.3$ & $6.1 \pm 1.3$ \\
\hline
\end{tabular}

the male skin spectra showing that variations in chemical composition of the skin types occur although changes in a chemical species cannot be specified.

In the wavenumber region from 1185 to $1010 \mathrm{~cm}^{-1}$ the bands are assigned to different conformations of lipid chains [45]. The areas and the entire shape of the spectra are very similar for the human in vivo skin spectra but different in the in vitro pig ear skin spectra. In particular the $1120 \mathrm{~cm}^{-1}$ band is relatively very much weaker, whereas the $1080 \mathrm{~cm}^{-1}$ band is more intense in the pig ear spectra. The $1120 \mathrm{~cm}^{-1}$ band is assigned to lipids with a trans configuration [6] and thus pig ear skin contains fewer lipids in trans configuration than human male and female skin. No assignment of the band at $1080 \mathrm{~cm}^{-1}$ has been found in the literature concerning IR spectra. However, using the assignments given for the Raman spectra [45] a lipid band at $1080 \mathrm{~cm}^{-1}$ band is connected to lipids mainly in the gauche configuration. Assuming this interpretation for the $1080 \mathrm{~cm}^{-1}$ IR band reveals that pig ear skin has a higher content of lipids with a gauche configuration than human skin. When comparing male and female skin spectra, male tends to have a larger peak at $1120 \mathrm{~cm}^{-1}$ - and hence a larger amount of lipids in the trans configuration.

Following sequential tape stripping male and female skin spectra undergo very much the same changes: the intensity of the water band at $3400 \mathrm{~cm}^{-1}$ increases with an increasing number of tape strips removed, because the water content becomes higher [16]. After 2 strips the water content was slightly higher in male than in female skin (Fig. 4). The increase in the intensity of the $3400 \mathrm{~cm}^{-1}$ water band is slightly faster in the female than the male spectra. Hence, after 6 tape strips this water band has the same intensity in male and female spectra and after further tape stripping there seems to be on an average slightly more water in the female skin. The amide I and II bands around 1650 and $1540 \mathrm{~cm}^{-1}$ broadens with tape stripping. This broadening can be explained by an increasing amount of proteins with a $\beta$-sheet secondary structure in the lower layers of stratum corneum. Broadening could also reflect a change in the physical state of the skin [49] and the amount of $\beta$-sheet would then be overestimated. 
The relative amount of $\beta$-sheet proteins we have just given for skin (tape stripped twice) is thus slightly increased compared to our recently reported results based on skin that has not been tape stripped [23]. With an increasing number of strips the content decreases of lipids with a trans configuration, determined from the intensity decrease at $1120 \mathrm{~cm}^{-1}$. For pig ear skin spectra the tape stripping sequence does not induce nearly the same changes and only a strong increase of the water band is seen. This means that after removal of 16 tape strips male and female spectra has become more alike whereas the pig ear skin is still very different to the human skin.

\subsection{Chemometrics}

PCA models were performed to give a better view of how each spectrum correlates to the others in the entire spectral range instead of just looking at average values at selected wavenumbers like we did with the curve fitting results. PLS models were performed to correlate the found differences to species or gender and to test the classification accuracy.

\subsubsection{NIR-FT-Raman spectra}

In the PCA model on Raman spectra of male and female skin in vivo and pig ear skin in vitro wavenumbers from 2700 to $1800 \mathrm{~cm}^{-1}$ were eliminated because no spectral information is found here. Wavenumbers below $900 \mathrm{~cm}^{-1}$ were also kept out of the model because the fiber optic probe gives rise to some peaks from quartz with varying intensities. The scoreplot of PC1 versus PC2 for all skin spectra is given in Fig. 5. In this plot pig ear skin is clearly differentiated from the human skin and in agreement with the curve fitting results pig ear and female spectra differs the most and male spectra are found somewhere
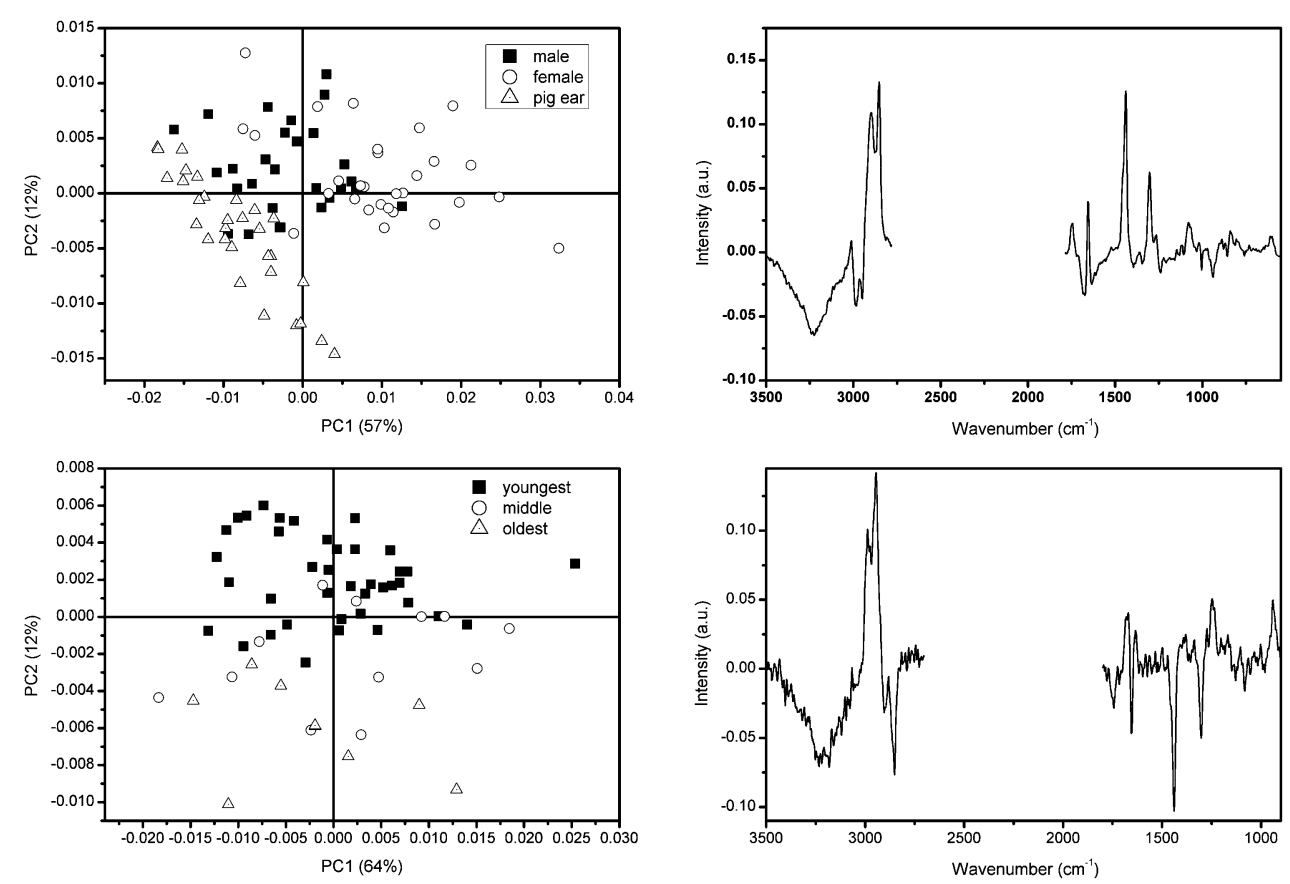

Fig. 5. (Top row) Scoreplot of PC1 vs. PC2 for Raman spectra of male and female skin in vivo and pig ear skin in vitro (left) and the corresponding loading of PC1 (right). (Bottom row) Scoreplot of PC1 vs. PC2 for Raman spectra of male and female skin in vivo (left). Subjects are marked by age and divided into groups of the youngest (20-34 years), middle-aged (35-49 years) and oldest (50-65 years). Corresponding loading of PC2 (right). 
between them. The loading of PC1, which is given in Fig. 5, is almost a pure lipid spectrum showing a higher contribution from lipids in the female skin and a higher contribution from water in the pig ear skin spectra.

Performing PCA on male and female spectra alone clearly shows a tendency of separation with respect to gender in the first PC: $78 \%$ of the female spectra are given positive PC1 values whereas $82 \%$ of the male spectra are given negative values. The loading of PC1 is very much the same as for the PCA of all spectra (Fig. 5), and hence shows that female skin contains more lipids whereas male skin have a higher content of water. PC2 correlates to the age of the volunteers (Fig. 5). The PC2 loading (Fig. 5) shows an increase in both water and lipids with age while the protein content is decreased or the structure is changed. This correlates well with the previously discussed difference in skin thickness which is expected to decrease with age [50], although it has been stated that the skin maintains its thickness up to the seventh decade [48]. However, a difference or change in skin thickness still does not cover for all of the spectral differences - especially since water and lipids both increase with age: a decrease in skin thickness would only increase the lipid content in the spectra with respect to an increased contribution from subcutaneous fat.

In addition, PCA and PLS models were performed on the dataset of calculated areas from the curve fitting procedure, of which the mean values are given in Table 1 . The areas were calculated for normalized spectra and hence no pretreatment was necessary to perform the models. The variables were weighted with $1 / \operatorname{Sdev}$ (standard deviation) to get them in the same range and let them contribute equally to the model, which would otherwise be dominated by the amide I band areas. In the PCA model on calculated areas from all human and pig ear skin spectra, pig ear in vitro and female in vivo skin samples are completely separated in the first PC, whereas male in vivo skin samples are found in between (Fig. 6). The corresponding loadings (Fig. 6) show that the areas defining the female spectra are from the peaks at $1300,2885,2854,1654,1750$ and $1270 \mathrm{~cm}^{-1}$, which are all characteristic of lipids. The $1654 \mathrm{~cm}^{-1}$
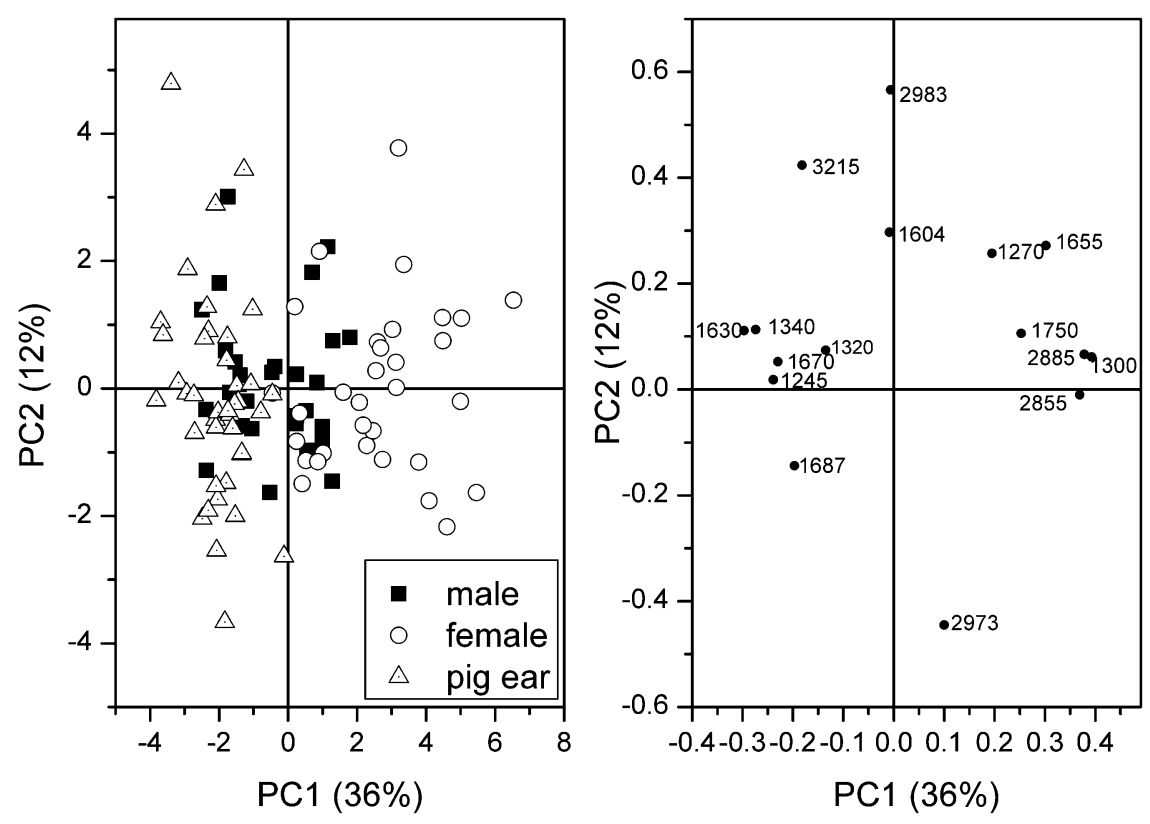

Fig. 6. (Left) Scoreplot of PC1 vs. PC2 from PCA of calculated areas in Raman spectra of male and female skin in vivo and pig ear skin in vitro. (Right) Corresponding loadings plot of PC1 vs. PC2. 
band is predominantly from the $\alpha$-helical keratines but also has some contribution from the amide I mode of the ceramides, which is one of the primary lipid classes [5]. The areas defining the pig ear skin spectra are from the peaks assigned to water $\left(3215 \mathrm{~cm}^{-1}\right), \beta$-sheets or collagen in general $(1630,1670$ and $\left.1685 \mathrm{~cm}^{-1}\right)$ or proteins specified to the epidermis $\left(1345\right.$ and $\left.1320 \mathrm{~cm}^{-1}\right)$ or dermis $\left(1245 \mathrm{~cm}^{-1}\right)$.

In the PLS model of human skin in vivo, discrimination was made by assigning male subjects to class 0 and female subjects to class 1 . Interpretation of the prediction is simple, as a predicted value below 0.5 means male and a predicted value above 0.5 means female. Only the first component seems to be related to gender and the corresponding loadings of the $x$-variables are very much the same as just described for the PCA of all spectra, except that the band at $1270 \mathrm{~cm}^{-1}$ does not have much influence on the model. The model correctly predicts $89 \%$ of the female skin samples and $83 \%$ of the males.

In the PLS model of male skin in vivo and pig ear skin in vitro discrimination was again made by assigning male subjects to class 0 and pig ear samples to class 1 . Hence, a predicted value below 0.5 means male and a predicted value above 0.5 means pig ear. The scoreplot shows a high degree of separation, and the corresponding loadings are primarily based on the areas of the lipid characteristic peaks at 1300, 2884 and $2850 \mathrm{~cm}^{-1}$ (male) and the protein characteristic peaks at 1344, 1670, 1630 and $1245 \mathrm{~cm}^{-1}$ (pig ear). Using 1 component the model correctly predicts $75 \%$ of the male subjects and $98 \%$ of the pig ears.

The first two loadings in the PLS model of female skin in vivo and pig ear skin in vitro are essentially the same as given in Fig. 6 for the PCA on all skin spectra. Using 2 components the model correctly predicts $97 \%$ of the female subjects and $100 \%$ of the pig ear skin samples.

\subsubsection{FT-NIR-spectra}

In several of the human skin spectra, all incident NIR light is absorbed around $5180 \mathrm{~cm}^{-1}$ and hence the $5180 \mathrm{~cm}^{-1}$ water band cannot be used for modeling. Hence this band is eliminated in the PCA model of the NIR spectra. The PCA of the NIR spectra (Fig. 7) even more clearly differentiates the pig ear in vitro skin spectra from the human in vivo skin spectra. However, male skin spectra still get scores closer to those of the pig ear skin than does the female skin spectra. The loading of PC1 is given in Fig. 7 and shows very much the same peaks as the recorded NIR spectra. However, the relative intensities are not the same in the loading as in the original spectra. The lipid bands at 8220,5780 and $5675 \mathrm{~cm}^{-1}$ are much more intense in the loading than in the skin spectra compared to the intensity of the water band at $6900 \mathrm{~cm}^{-1}$. Hence, the loading shows that human skin contains a higher amount of lipids and also that the amount or bonding of the water differs for human in vivo and pig ear in vitro skin. Making the PCA on male and female spectra alone further separates the two genders: based on the first PC alone, $67 \%$ of the female spectra are given positive values whereas $77 \%$ of the male spectra are given negative values (Fig. 7). The loading of PC1 (not shown) is very much the same as for the PCA of all spectra (Fig. 7) except that the water band at $6900 \mathrm{~cm}^{-1}$ is even less intense in this loading whereas the intensity of lipid bands is further increased. Hence this loading shows that the separation of male and female spectra is mainly due to the lipid content of the skin. The third PC shows some dependency on age and the spectra of the oldest participants are given positive PC3 scores whereas the youngest are mainly given negative scores (Fig. 7).

\subsubsection{ATR-FTIR spectra}

In the PCA model of the ATR-FTIR spectra recorded after removal of 2 tape strips, spectra of pig ear skin in vitro are again completely separated from human skin with male PC1 scores lying between the scores of females and pig ears (Fig. 8). The corresponding PC1 loading is almost sufficient to perform the separation as it spans $75 \%$ of the variation in the data set. This loading shows that the pig ear skin has 

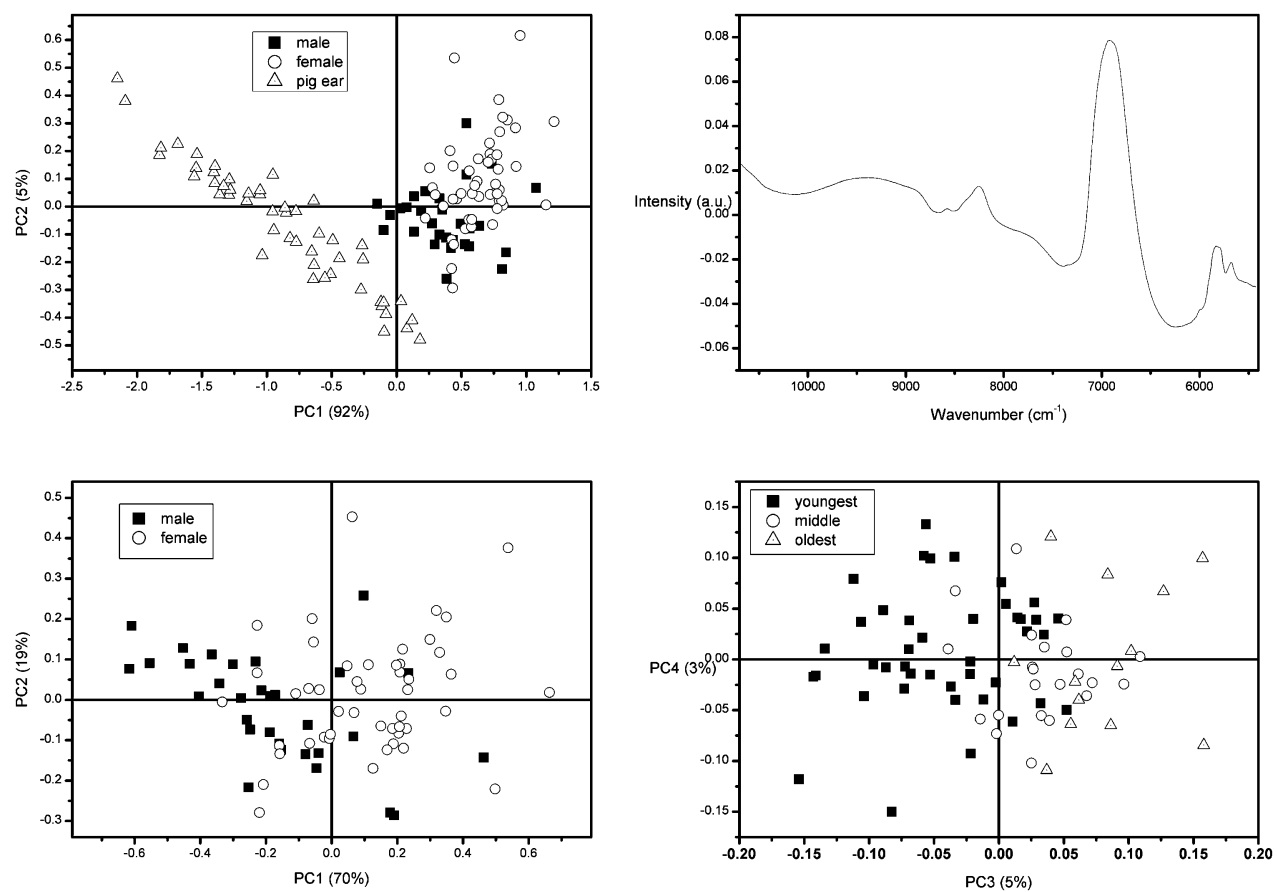

Fig. 7. (Top row) Scoreplot of PC1 vs. PC2 for NIR spectra of male and female skin in vivo and pig ear skin in vitro (left) and the corresponding loading of PC1 (right). (Bottom row) Scoreplots from PCA for NIR spectra of male and female skin in vivo. (Left) PC1 vs. PC2; subjects marked by gender. (Right) PC3 vs. PC4; subjects marked by age and divided into groups of the youngest (20-35 years), middle-aged (36-50 years) and oldest (51-65 years).
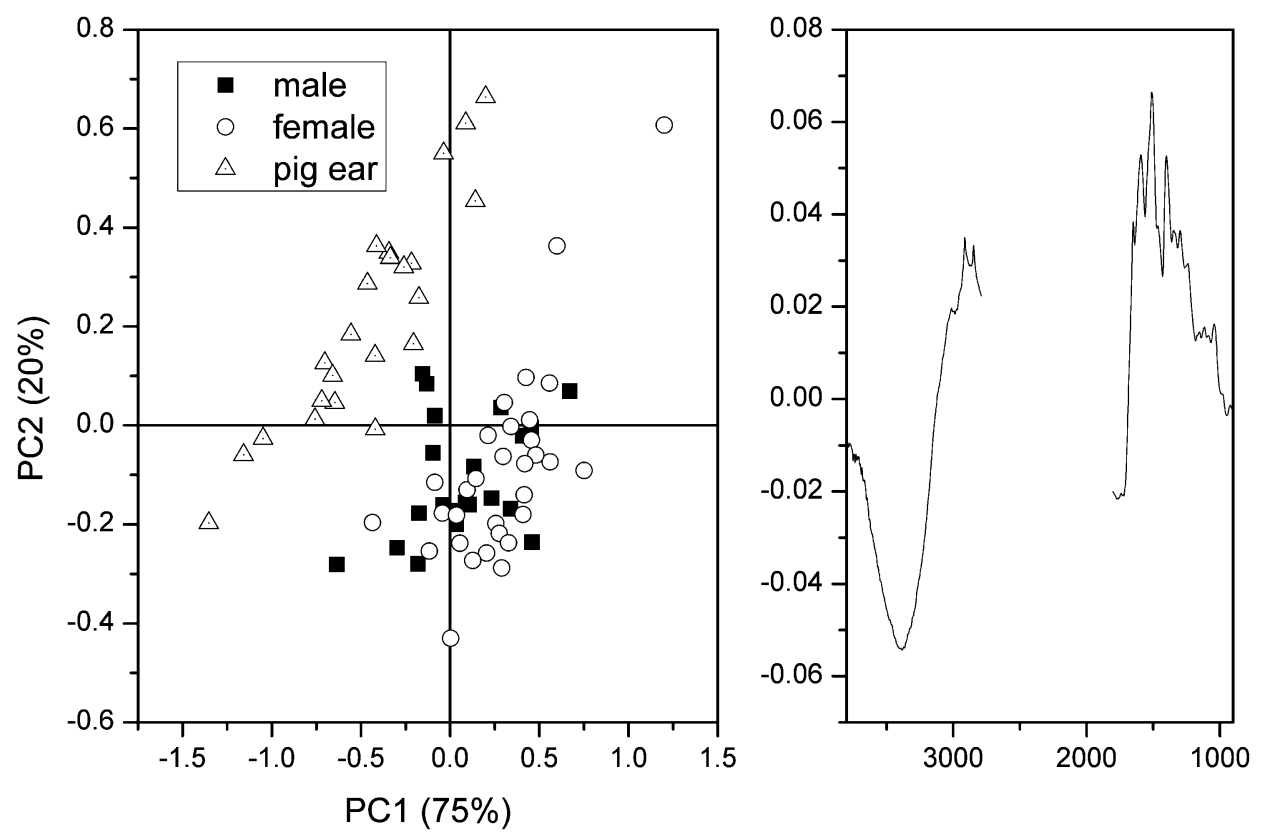

Fig. 8. (Left) Scoreplot of PC1 vs. PC2 for ATR-FTIR spectra of male and female skin in vivo and pig ear skin in vitro - spectra are recorded after removal of 2 tape strips. (Right) Loading of PC1. 
a higher content of water - given by the peak at $3200 \mathrm{~cm}^{-1}$ - whereas the human skin contains a higher amount of lipids - given by the peaks at 2920, 2850, 1400 and $1030 \mathrm{~cm}^{-1}$. Based on the PC1 loading the protein structure may vary as well, as the relative intensities of the amide I and II bands differ from the recorded spectra and hence does not correspond to the overall contribution from proteins in the spectra. The separation of pig ear and human skin is also found for the spectra recorded prior to tape stripping as well as for the spectra recorded at any given step in the tape stripping sequence. This separation with respect to species or in vivo/in vitro differences is increased with the number of tape strips. In Fig. 9 is given the scoreplots of the first two PCs in the PCAs made on the recorded spectra of human skin in vivo at the first 8 steps of the tape stripping sequence. Prior to tape stripping gender is not related to the greatest variation in the recorded spectra and separation only occurs in the fourth PC (not shown). In the PCA on spectra recorded after removal of 1 tape strip the second PC seems to be slightly related to gender since $68 \%$ of the females get positive PC2 scores and $71 \%$ of the males are given negative scores. In the PCAs on spectra recorded after 2-8 tape strips the same $71 \%$ of the male spectra is consistently getting negative scores whereas the female spectra are assigned to positive scores for 56-68\% of the samples - with the separation getting weaker with the number of tape strips. In the PCA models made on spectra recorded after removal of 10-16 tape strips no separation with respect to gender is seen. This lack of separation may well be explained by the before mentioned inter personal differences in sensitivity towards the tape stripping procedure. However, when focusing on the lipid bands in the $1185-1010 \mathrm{~cm}^{-1}$ region, the gender separation is regained as $65 \%$ of the female subjects are given negative PC1 scores and $62 \%$ of the male subjects are assigned positive scores. The corresponding loading shows a higher intensity of the $1118 \mathrm{~cm}^{-1}$ peak in the male spectra revealing that there are more lipids in the male skin in the trans conformation. Making a PCA model on all of the recorded male and female in vivo skin spectra - following the sequential tape stripping procedure - the spectra recorded after removal of none or a few tape strips are separated from those recorded after removal of several tape strips. The primary part of the separation lies in the first PC and the corresponding loading shows that the majority of change is the increase of water with the number of strips, whereas all other peaks are apparently more intense before tape stripping (Fig. 10). This goes especially for the $\mathrm{CH}$ stretching vibration bands from lipids at 2920 and $2850 \mathrm{~cm}^{-1}$ and the amide III band at $1245 \mathrm{~cm}^{-1}$ : the relative intensity of these bands are increased in the loading compared to the recorded spectra and hence may have a relatively larger variation following the sequential tape stripping. The scoreplot further shows that inter and intra gender differences are decreased with the number of tape strips as the samples are much more widespread prior to tape stripping than after.

PLS models were performed on the data set of calculated areas from the curve fitting procedure. The average values of the relative areas are given in Table 2. In the chemometric models the numeric areas are used as the MSC pretreatment corrects for the general intensity variations. Like for the Raman spectra, the variables were weighted with $1 / \operatorname{Sdev}$ to get them in the same range and let them contribute equally to the model, which would otherwise be dominated by the amide I band areas. Discrimination was again made by assigning male subjects to class 0 and pig ear samples to class 1 . In the scoreplot of the PLS regression on male in vivo and pig ear in vitro skin spectra recorded after removal of 2 tape strips, the male and pig ear samples are completely separated in the first 2 components (Fig. 11). The loading of the first component (Fig. 11) is spanned by the areas of the peaks at 1338, 1119, 1400, 1297, 1338, 1047 and $1120 \mathrm{~cm}^{-1}$ (important in human skin spectra) and the areas of the peaks at 1170, 1676, 1157, 1453, 1648, 1664 and $1032 \mathrm{~cm}^{-1}$ (important in pig ear skin spectra). The peaks at both poles of the loading are assigned to several different skin constituents and hence no further correlations can be specified. The loading shows no correlation between the $1620 \mathrm{~cm}^{-1}$ amide I band of $\beta$-keratins and the 1244 amide III 

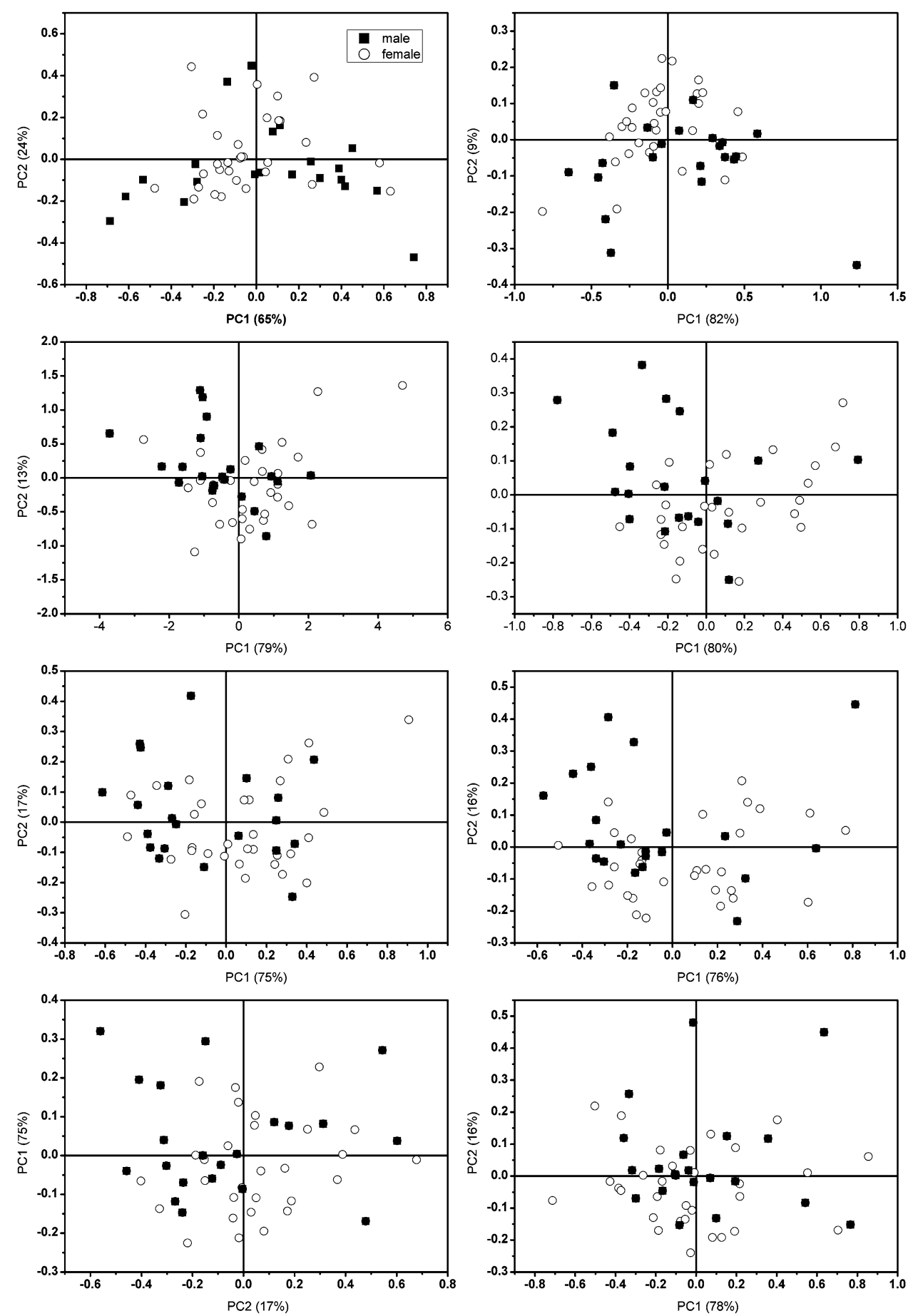

Fig. 9. Scoreplot of PC1 vs. PC2 for ATR-FTIR spectra of male and female in vivo skin spectra. Spectra are recorded after removal of $0,1,2,3,4,6,8$ and 10 tape strips, respectively - starting at 0 in the upper left corner and going from left to right. 


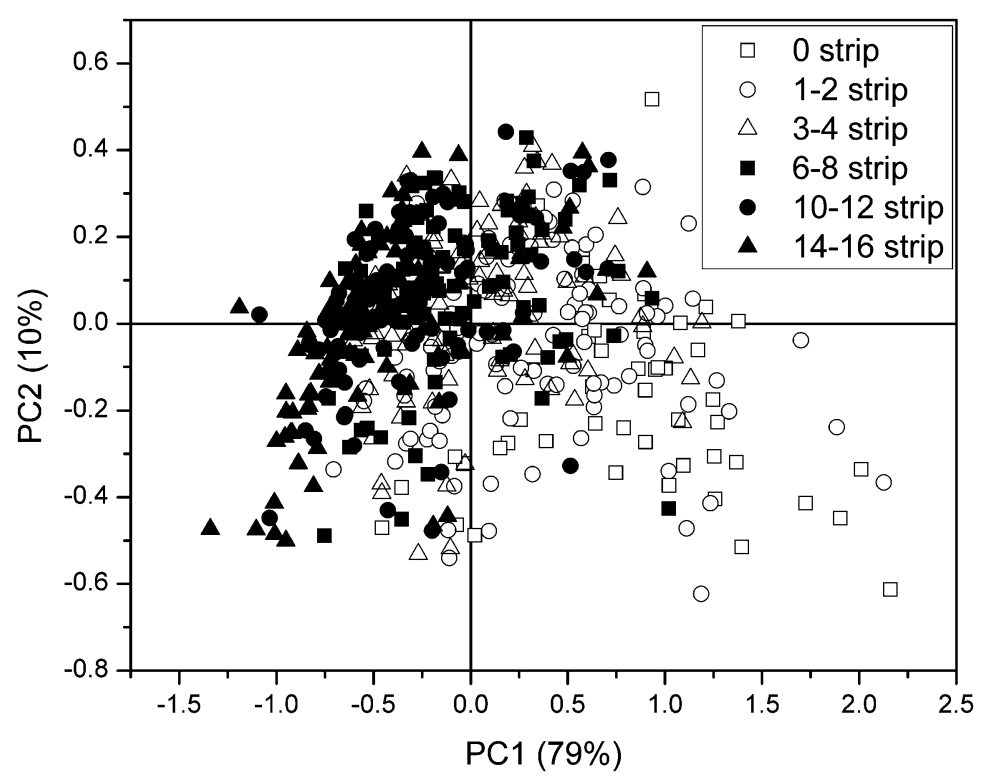

Fig. 10. Scoreplot of PC1 vs. PC2 for ATR-FTIR spectra of human skin in vivo following every second step of the tape stripping sequence.
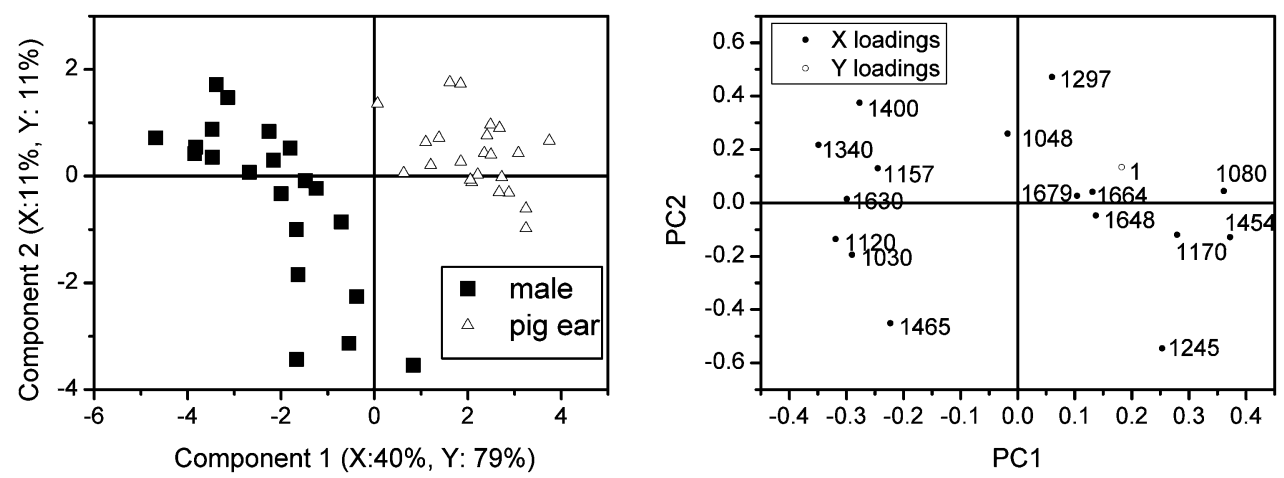

Fig. 11. Scoreplot (left) and loadings plot (right) of component 1 vs. component 2 from PLS-DA of calculated areas in ATR-FTIR spectra of male skin in vivo and pig ear skin in vitro, recorded after removal of 2 tape strips.

band as the former is characteristic of male spectra whereas the latter is characteristic of pig ear skin spectra. Thus males tend to have a higher content of keratins with a $\beta$-sheet secondary structure, which could not be read from the relative areas in Table 2. Using 2 components the model correctly predicts $100 \%$ of both male and pig ear skin samples. A PLS discriminant analysis performed on female and pig ear skin spectra showed essentially the same scores and loadings as those just described for males versus pig ears, and this model also $100 \%$ correctly predicts the species of all samples.

In the PLS model on all human in vivo spectra discrimination was made by assigning female subjects to class 0 and male samples to class 1 . In the scoreplot of component 1 versus component 2, male and female subjects are largely separated (Fig. 12). The corresponding loadings (Fig. 12) show that the female spectral characteristics are primarily made up by the areas of the 1338, 1297 and $1400 \mathrm{~cm}^{-1}$ bands. Hence it seems reasonable that these peaks are correlated to the same lipid classes, which gives 

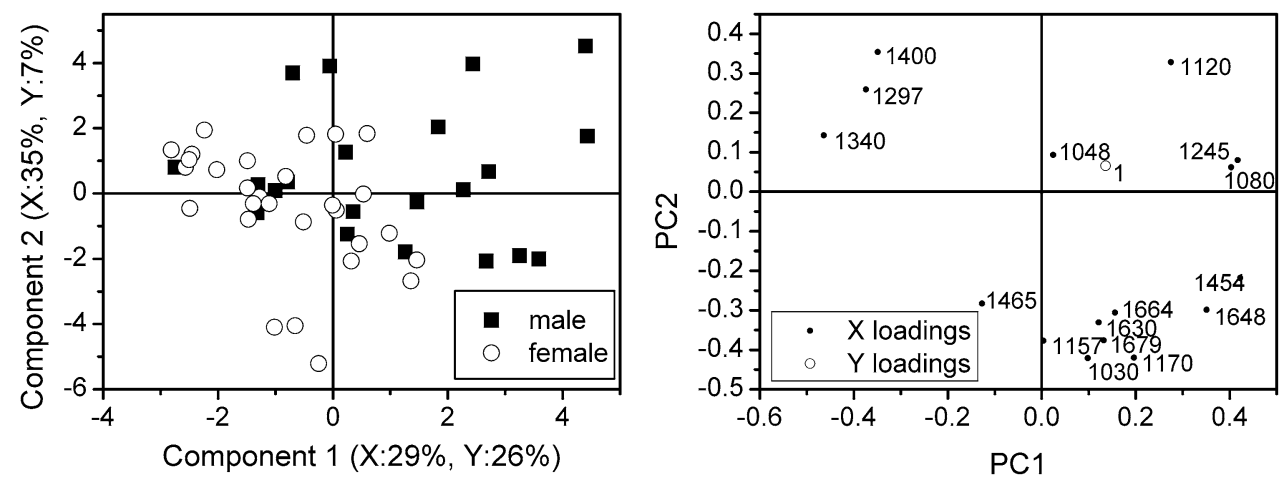

Fig. 12. Scoreplot (left) and loadings plot (right) of component 1 vs. component 2 from PLS-DA of calculated areas in ATR-FTIR spectra of male and female skin in vivo recorded after removal of 2 tape strips.

a new assignment of the so far unassigned $1338 \mathrm{~cm}^{-1}$ band. The PLS discriminant analysis correctly classifies $64 \%$ of the male subjects and $93 \%$ of the females.

\section{Conclusion}

The combination of near-FT-Raman, FT-NIR and ATR-FTIR spectroscopy on human skin in vivo and pig ear skin in vitro reveals information about the composition of different layers in the skin. With all of the spectroscopic techniques differences and similarities were found between spectra of pig ear skin in vitro and human skin in vivo and also between male and female skin.

Signals in the near-FT-Raman spectra are predominantly coming from the deeper layers of the skin and some of the described spectral differences may well reflect a difference in skin thickness. We found that male skin and pig ear skin have the same thicknesses whereas female skin is slightly thinner. Male skin in vivo and pig ear skin in vitro was found to have similar contents of water whereas males had higher lipid content and pig ear skin contained a higher amount of $\beta$-keratin or collagen. Besides from the difference in skin thicknesses for male and female skin in vivo we found that female skin contains more lipids and more water whereas male skin contains more $\beta$-keratin or collagen relative to skin thickness. That is, though the overall spectral contribution from water was higher in male than female skin, it does not compare to the increased thickness of the skin and thus female skin contains relatively more water.

Signals in the FT-NIR spectra arise from the epidermal skin layer where the primary spectral differences were found with respect to bands assigned to lipids. The NIR spectra corroborated the Raman spectroscopic results in showing that female skin has the highest content of lipids and pig ear skin has the lowest lipid content. The NIR spectra showed no differences with respect to skin proteins, thus suggesting that the differences in the amount of non- $\alpha$-helical proteins found in the Raman spectra, can be assigned to the dermal layers of the skin; i.e. collagens.

The ATR-FTIR spectra, which were recorded following sequential tape stripping, reveals information about the outermost layers of the skin within a few microns from the skin surface. These spectra shows that water content for all skin types increases in the lower layers of SC, though pig ear skin in vitro contains more water than human skin at any layer. Male skin contains more water than female in the outermost layers of SC but the increase is smaller and thus female skin tends to have a slightly higher content of water in the lower layers. Further, differences were found with respect to conformation of lipid chains. Female skin tends to contain a higher amount of lipids but this could not be assigned to 
specific configurations. Pig ear skin contains the highest content of lipids with a gauche configuration whereas male skin shows a higher content of lipids with a trans conformation.

The PCA and PLS models showed an almost complete separation of pig ear skin in vitro and human skin in vivo, and also gave a 65-93\% separation with respect to gender in all layers of the skin. Further, the PCA models on Raman and NIR spectra, respectively, showed a dependency of age where the spectral intensities of bands from lipids and water was increased with age.

\section{Acknowledgements}

This study was accepted by the Danish National Committee on Biomedical Research Ethics. We thank the Danish Agency for Science, Technology and Innovation for a PhD grant to TMG. We thank Rasmus Bro (Department of Food Science, University of Copenhagen), who has been very helpful in the chemometric treatment of the results.

\section{References}

[1] H. Schaefer and T.E. Redelmeier, Skin Barrier - Principles of Percutaneuos Absorption, Karger, New York, 1996.

[2] H.P. Baden and L. Bonar, The $\alpha$-fibrous proteins of epidermis, Journal of Investigative Dermatology 51 (1968), 478-483.

[3] B.G. Frushour and J.L. Koenig, Raman scattering of collagen, gelatin and elastin, Biopolymers 14 (1975), 379-391.

[4] B.W. Barry, H.G.M. Edwards and A.C. Williams, Fourier transform Raman and infrared vibrational study of human skin: Assignment of spectral bands, Journal of Raman Spectroscopy 23 (1992), 641-645.

[5] M. Wegener, R. Neubert, W. Rettig and S. Wartewig, Structure of stratum corneum lipids characterized by FT-Raman spectroscopy and DSC. I. Ceramides, International Journal of Pharmaceutics 128 (1996), 203-213.

[6] G.W. Lucassen, P.J. Caspers and G.J. Puppels, In vivo infrared- and Raman spectroscopy of human stratum corneum, Proceedings of SPIE 3257 (1998), 52.

[7] P.J. Caspers, G.W. Lucassen, R. Wolthius, H.A. Bruining and G.J. Puppels, In vitro and in vivo Raman spectroscopy of human skin, Biospectroscopy 4 (1998), 31-39.

[8] O.F. Nielsen, C. Johansson, K.L. Jakobsen, D.H. Christensen, M.R. Wiegell, T. Pedersen, M. Gniadecka, H.C. Wulf and P. Westh, Water structure and water/protein interactions in biological materials characterized by Raman spectroscopy, in: Optical Devices and Diagnostics in Materials Science, Proceedings of SPIE, Vol. 4098, D.L. Andrews, T. Asakura, S. Jutamulia, W.P. Kirk, M.G. Lagally, R.B. Lal and J.D. Trolinger, eds, Bellingham, WA, 2000, pp. 160-168.

[9] P.J. Caspers, G.W. Lucassen, E.A. Carter, H.A. Bruining and G.J. Puppels, In vivo confocal Raman microspectroscopy of the skin: Noninvasive determination of molecular concentration profiles, Journal of Investigative Dermatology 116 (2001), 434-442.

[10] H.G.M. Edwards, A.C. Williams and B.W. Barry, Potential application of FT-Raman spectroscopy for dermatological diagnostics, Journal of Molecular Structure 347 (1995), 379-388.

[11] M. Gniadecka, O.F. Nielsen and H.C. Wulf, Water content and water structure in malignant and benign skin tumors, Journal of Molecular Structure 661 (2003), 405-410.

[12] M. Osada, M. Gniadecka and H.C. Wulf, Near-infrared Fourier transform Raman spectroscopic analysis of proteins, water and lipids in intact normal stratum corneum and psoriasis scales, Experimental Dermatology 13 (2004), 391-395.

[13] N.S. Eikje, Y. Ozaki, K. Aizawa and S. Arase, Fiber optic near-infrared Raman spectroscopy for clinical noninvasive determination of water content in deseased skin and assessment of cutaneous edema, Journal of Biomedical Optics 101 (2005), 1-13.

[14] A.C. Williams, B.W. Barry and H.G.M. Edwards, Comparison of Fourier transform Raman spectra of mammalian and reptilian skin, Analyst 119 (1994), 563-566.

[15] T.M. Greve, N. Rastrup Andersen, K. Birklund Andersen, M. Gniadecka, H.C. Wulf and O. Faurskov Nielsen, Biomedical aspects of water structure in human and animal skin: A near infrared Fourier transform Raman study, in: New Approaches in Biomedical Spectroscopy, ACS Symposium Series, Vol. 963, K. Kneipp, R. Aroca, H. Kneipp and E. Wentrup-Byrne, eds, American Chemical Society, Washington, DC, 2007, pp. 30-40. 
[16] G.W. Lucassen, P.J. Caspers and G.J. Puppels, Water content and water profiles in skin measured by FTIR and Raman spectroscopy, Proceedings of SPIE 4162 (2000), 39-45.

[17] L. Brancaleon, M.P. Bamberg, T. Sakamaki and N. Kollias, Attenuated total reflection Fourier transform infrared spectroscopy as a possible method to investigate biophysical parameters of stratum corneum in vivo, Journal of Investigative Dermatology 116 (2001), 380-386.

[18] J.E. Harrison, A.C. Watkinson, D.M. Green, J. Hadgraft and K. Brain, The relative effect of Azone and Transcutol on permeant diffusivity and solubility in human stratum corneum, Pharmaceutical Research 13 (1996), 542-546.

[19] Y. Raichlin, I. Goldberg, S. Brenner, E. Schulzinger and A. Katzir, Infrared fiberoptic evanescent wave spectroscopy for the study of diffusion in the human skin, Proceedings of SPIE 4614 (2002), 101-108.

[20] J.-C. Tsai, C.-Y. Lin, H.-M. Sheu, Y.-L. Lo and Y.-H. Huang, Noninvasive characterization of regional variation in drug transport into human skin, Pharmaceutical Research 20 (2003), 632-638.

[21] C. Curdy, A. Naik, Y.N. Kalia, I. Alberti and R.H. Guy, Noninvasive assessment of the effect of formulation excipients on stratum corneum barrier function in vivo, International Journal of Pharmaceutics 271 (2004), 251-256.

[22] A. Carden, M.G. Yost and R.A Fenske, Noninvasive method for the assessment of dermal uptake of pesticides using attenuated total reflectance infrared spectroscopy, Applied Spectroscopy 59 (2005), 293-299.

[23] T.M. Greve, K.B. Andersen and O.F. Nielsen, Penetration mechanism of dimethyl sulfoxide in human and pig ear skin An ATR-FTIR and Near-FT Raman spectroscopic in vivo and in vitro study, Spectroscopy: An International Journal, in press.

[24] K.A. Martin, Direct measurement of moisture in skin by NIR spectroscopy, Journal of the Society of Cosmetical Chemists 44 (1993), 249-261.

[25] K. Martin, In vivo measurements of water in skin by near-infrared reflectance, Applied Spectroscopy 52 (1998), 10011007.

[26] E.M. Attas, M.G. Sowa, T.B. Posthumus, B.J. Schattka, H.H. Mantsch and S.L. Zhang, Near-IR spectroscopic imaging for skin hydration: The long and short of it, Biopolymers 67 (2002), 96-106.

[27] H. Arimoto, M. Egawa and Y. Yamada, Depth profile of diffuse reflectance near-infrared spectroscopy for measurement of water content in skin, Skin Research and Technology 11 (2005), 27-35.

[28] E.-J. Suh, Y.-A. Woo and H.-J. Kim, Determination of water content in skin by using a FT neat infrared spectrometer, Archives of Pharmaceutical Research 28 (2005), 458-462.

[29] Y.-A. Woo, J.-W. Ahn, I.-K. Chun and H.-J. Kim, Development of a method for the determination of human ski moisture using a portable near-infrared system, Analytical Chemistry 73 (2001), 4964-4971.

[30] M. Egawa, H. Arimoto, T. Hirao, M. Takahashi and Y. Ozaki, Regional difference of water content in human skin studied by diffuse-reflectance near-infrared spectroscopy: Consideration of measurement depth, Applied Spectroscopy 60 (2006), 24-28.

[31] P. Geladi, J. Nyström, J.W. Eriksson and A. Nilsson, A multivariate NIR study of skin alterations in diabetic patients as compared to control subjects, Journal of Near Infrared Spectroscopy 8 (2002), 217-227.

[32] R.K. Lauridsen, H. Everland, L.F. Nielsen, S.B. Engelsen and L. Nørgaard, Exploratory multivariate spectroscopic study on human skin, Skin Research and Technology 9 (2003), 137-146.

[33] J.W. Wiechers, M. Sneider, N.A.G. Dekker and W.G. Hansen, Factors influencing skin moisturization signal using nearinfrared spectroscopy, IFSCC Magazine 6 (2003), 19-26.

[34] E. Widjaja, G.H. Lim and A. An, A novel method for human gender classification using Raman spectroscopy of fingernail clippings, Analyst 133 (2008), 493-498.

[35] N. Sekkat, Y.N. Kalia and R.H. Guy, Biophysical study of porcine ear skin in vitro and its comparison to human skin in vivo, Journal of Pharmaceutical Sciences 91 (2002), 2376-2381.

[36] D. Bommannan, R.O. Potts and R. Guy, Examination of stratum corneum barrier function in vivo by infrared spectroscopy, Journal of Investigative Dermatology 95 (1990), 403-408.

[37] R. Mendelsohn, C.R. Flach and D.J. Moore, Determination of molecular conformation and permeation in skin via IR spectroscopy, microscopy, and imaging, Biochimica et Biophysica Acta 1758 (2006), 923-933.

[38] A.M. Kligman and E. Christophers, Preparation of isolated sheets of human stratum corneum, Archives of Dermatology 88 (1963), 702-705.

[39] N.J. Harrick, Internal Reflection Spectroscopy, Interscience, New York, 1967.

[40] R.J. Scheuplein, A survey of some fundamental aspects of the absorption and reflection of light by tissue, Journal of the Society of Cosmetic Chemists 15 (1964), 111-122.

[41] E. Dreassi, C. Ceramelli, L. Fabbri, F. Vocioni, P. Bartalini and P. Corti, Application of near-infrared reflectance spectrometry in the study of atopy: Part 1. Investigation of skin spectra, Analyst 122 (1997), 767-770.

[42] Y. Maeda and H. Kitano, The structure of water in polymer systems as revealed by Raman spectroscopy, Spectrochimica Acta Part A 51 (1995), 2433-2446.

[43] G.E. Walrafen and Y.C. Chu, Linearity between structural correlation length and correlated proton intensity from amorphous ice and supercooled water up to dense supercritical steam, Journal of Physical Chemistry 99 (1995), 11225-11229. 
[44] A.N.C. Anigbigu, A.C. Williams, B.W. Barry and H.G.M. Edwards, Fourier transform Raman spectroscopy of interactions between the penetration enhancer dimethyl sulfoxide and human stratum corneum, International Journal of Pharmaceutics 125 (1995), 265-282.

[45] A.T. Tu, Raman Spectroscopy in Biology: Principles and Applications, Wiley, New York, 1982.

[46] B. Stuart, Biological Applications of Infrared Spectroscopy, Wiley, New York, 1997.

[47] A.D. Meade, F.M. Lyng, P. Kneif and H.J. Byrne, Growth substrate induced functional changes elucidated by FTIR and Raman spectroscopy in in-vivo cultured human keratinocytes, Analytical and Bioanalytical Chemistry 387 (2007), 17171728.

[48] C. Escoffier, J. de Rigal, A. Rochefort, R. Vasselet, J.-L. Lévêque and P.G. Agache, Age-related mechanical properties of human skin. An in vivo study, Journal of Investigative Dermatology 93 (1989), 353-357.

[49] M. van de Weert, P.I. Haris, W.E. Hennink and D.J.A. Crommelin, Fourier transform infrared spectrometric analysis of protein conformation: Effect of sampling method and stress factors, Analytical Biochemistry 297 (2001), 160-167.

[50] S. Richard, B. Querleux, J. Bittoun, O. Jolivet, I. Idy-Peretti, O. de Lacherriere and J.-L. Leveque, Characterization of the skin in vivo by high resolution magnetic resonance imaging: water behaviour and age-related effects, Journal of Investigative Dermatology 100 (1993), 705-709. 


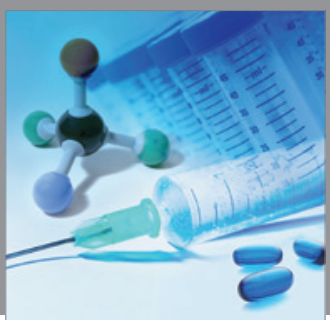

International Journal of

Medicinal Chemistry

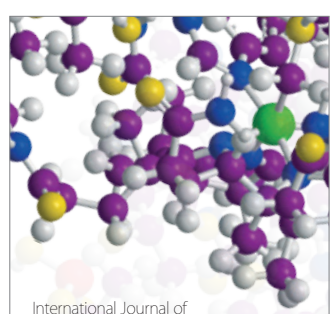

Carbohydrate Chemistry

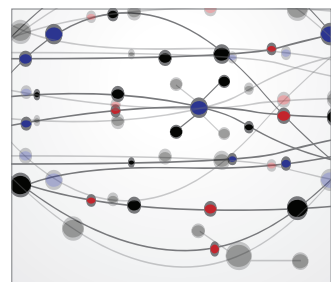

The Scientific World Journal
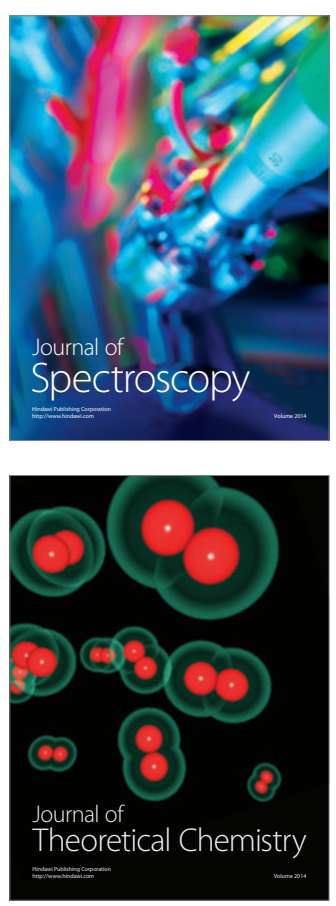
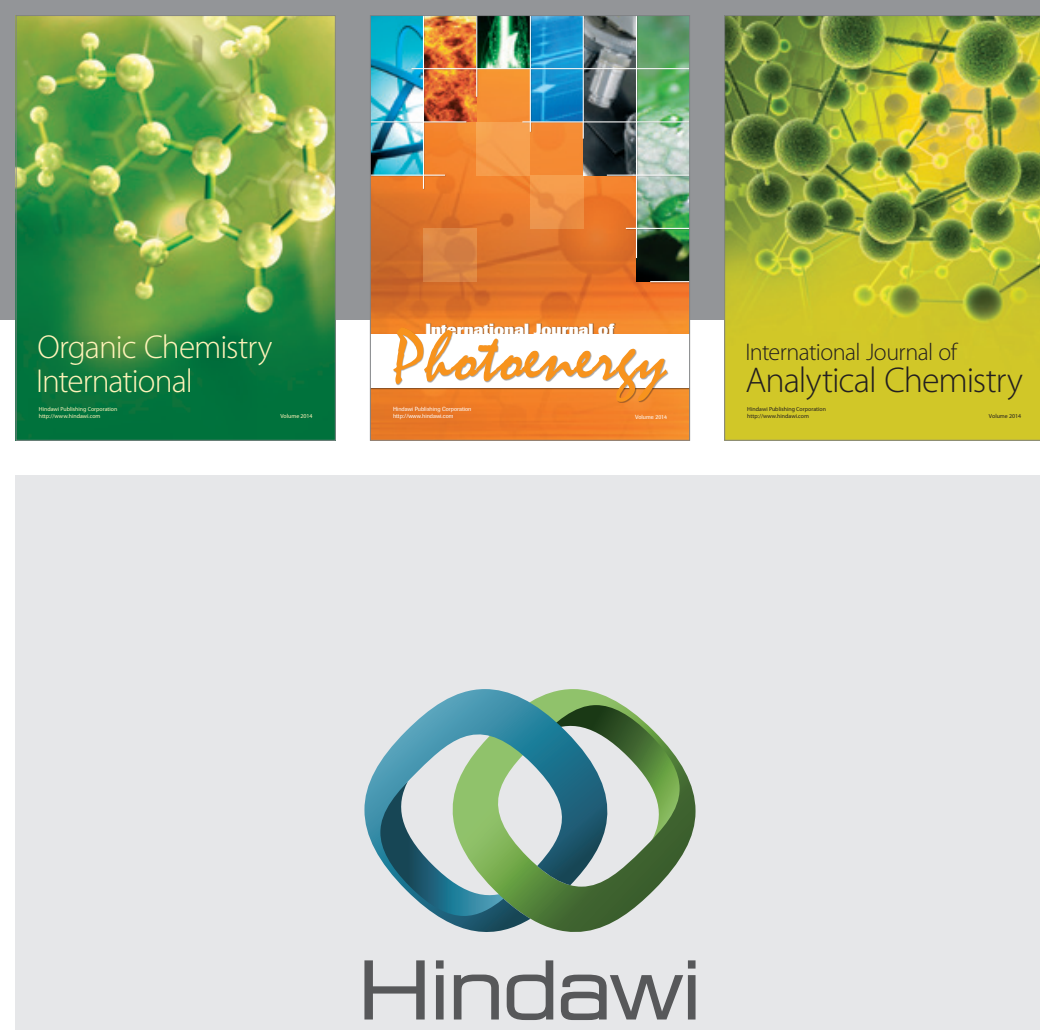

Submit your manuscripts at

http://www.hindawi.com
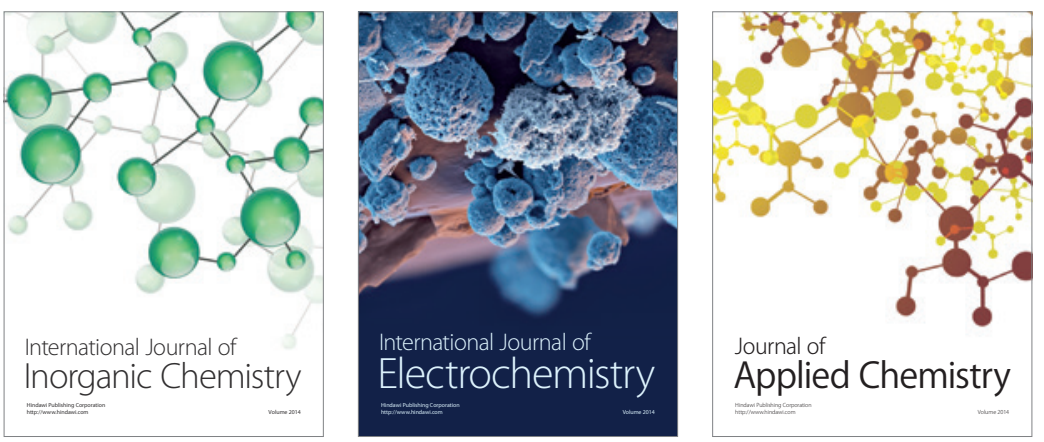

Journal of

Applied Chemistry
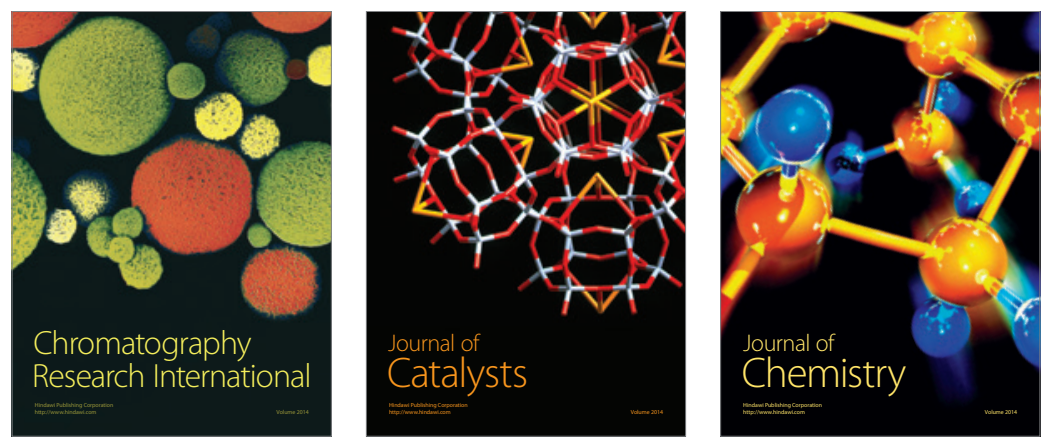
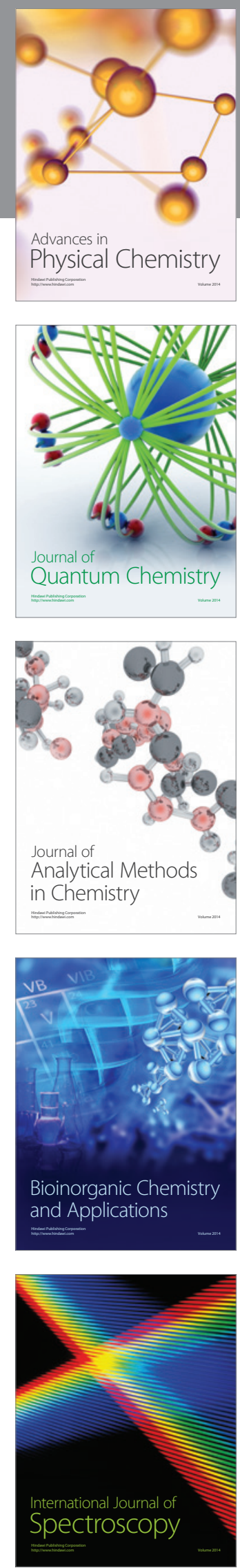